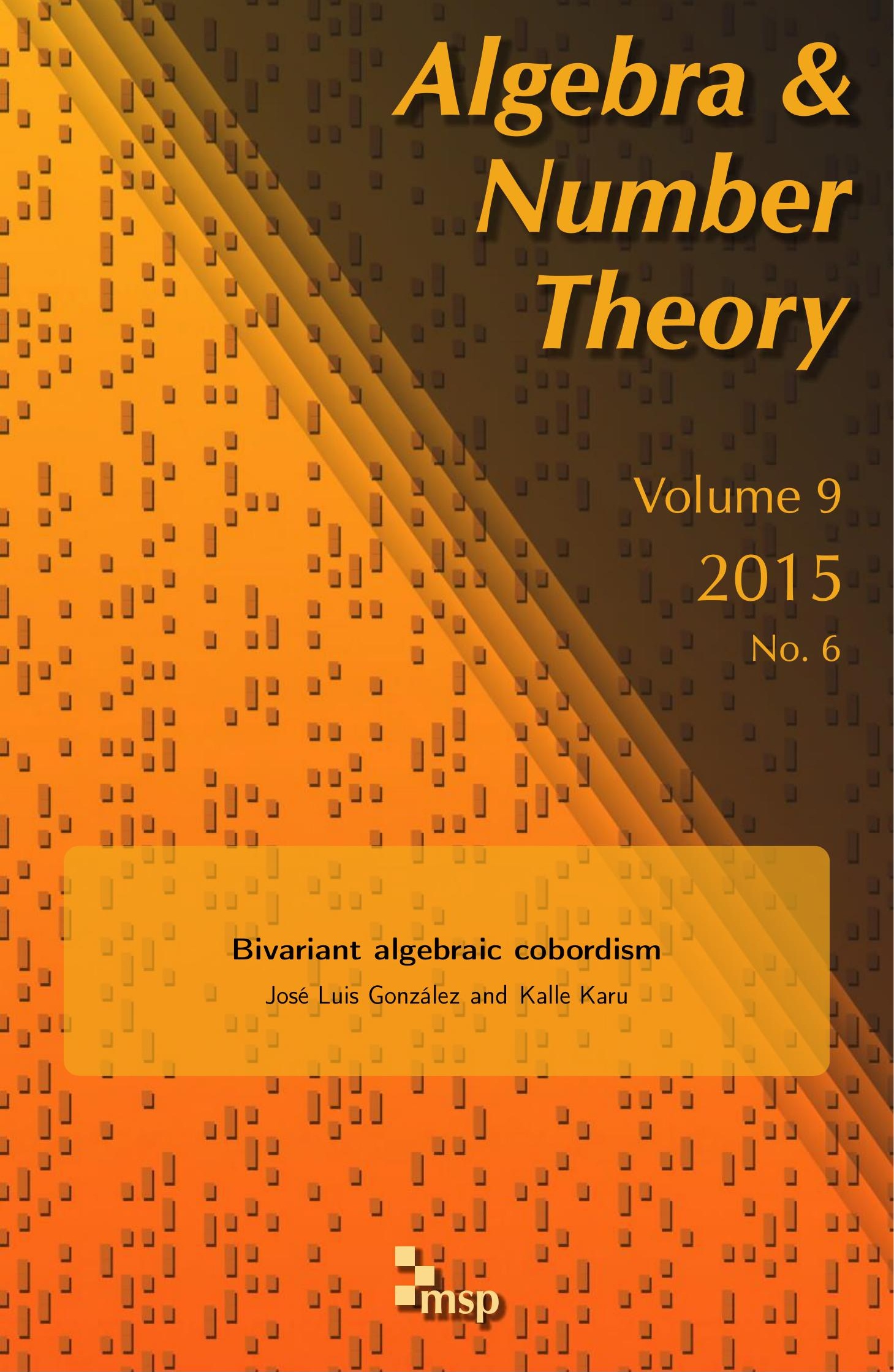




\title{
Bivariant algebraic cobordism
}

\author{
José Luis González and Kalle Karu
}

We associate a bivariant theory to any suitable oriented Borel-Moore homology theory on the category of algebraic schemes or the category of algebraic $G$ schemes. Applying this to the theory of algebraic cobordism yields operational cobordism rings and operational $G$-equivariant cobordism rings associated to all schemes in these categories. In the case of toric varieties, the operational $T$-equivariant cobordism ring may be described as the ring of piecewise graded power series on the fan with coefficients in the Lazard ring.

1. Introduction

1293

2. Refined oriented Borel-Moore prehomology theories

3. Operational bivariant theories

4. The equivariant version of an ROBM prehomology theory

5. Descent sequences

6. An overview of algebraic cobordism theory

7. Operational equivariant cobordism of toric varieties

References

\section{Introduction}

The purpose of this article is to study the operational bivariant theory $B^{*}$ associated to a refined oriented Borel-Moore prehomology theory $B_{*}$, and the equivariant versions of these theories. We apply this to the algebraic cobordism theory $\Omega_{*}$ of Levine and Morel [2007] to construct the operational bivariant cobordism theory $\Omega^{*}$. As an application, we describe the operational T-equivariant cobordism $\Omega_{T}^{*}\left(X_{\Delta}\right)$ for a quasiprojective toric variety $X_{\Delta}$.

Bivariant theories were defined in [Fulton and MacPherson 1981; Fulton 1998]. A bivariant theory assigns a group $B^{*}(X \rightarrow Y)$ to every morphism $X \rightarrow Y$ of schemes. The theory contains both a covariant homology theory $B_{*}(X)=B^{*}(X \rightarrow \mathrm{pt})$ and a contravariant cohomology theory $B^{*}(X)=B^{*}\left(\operatorname{Id}_{X}: X \rightarrow X\right)$, but the bivariant

This research was funded by NSERC Discovery and Accelerator grants. González thanks his coauthor and the University of British Columbia for their generosity during his postdoctoral position at UBC. MSC2010: primary 14C17; secondary 14C15, 14F43, 14M25, 55N22, 57R85.

Keywords: algebraic cobordism, bivariant and operational theories, operational (equivariant) cobordism, operational equivariant cobordism of toric varieties. 
theory can be more general in the sense that there may be invariants of the theory that are not determined by homology and cohomology alone [Fulton and MacPherson 1981]. It was also shown in [Fulton and MacPherson 1981; Fulton 1998] how to extend a given homology theory $B_{*}$ to a bivariant theory. Bivariant classes in this theory are certain compatible operators on the homology groups $B_{*}$; hence the bivariant theory is called the operational bivariant theory. The only bivariant theories we consider in this paper are the operational ones. An operational bivariant theory can be viewed as a method of constructing a cohomology theory $B^{*}$ out of a homology theory $B_{*}$. The cohomology theory takes values in rings, hence there is a well-defined intersection product in this theory. The Chern class operators naturally lie in the cohomology $B^{*}$.

The operational cohomology theory $B^{*}$ at first seems very intractable. A single element of $B^{*}(X)$ is defined by an infinite set of homomorphisms. However, Kimura [1992] has shown that, in case of Chow theory $A_{*}$, the bivariant cohomology groups $A^{*}(X)$ for an arbitrary variety $X$ can often be computed if one knows the homology groups $A_{*}(Y)$ for smooth varieties $Y$. Payne [2006] carried out this computation for the equivariant Chow cohomology $A_{T}^{*}$ of toric varieties. By a result of Brion [1997], the $T$-equivariant Chow ring $A_{T}^{*}\left(X_{\Delta}\right)$ of a smooth toric variety $X_{\Delta}$ can be identified with the group of integral piecewise polynomial functions on the fan $\Delta$. Payne showed that the ring of such functions on an arbitrary fan $\Delta$ gives the operational $T$-equivariant Chow cohomology $A_{T}^{*}\left(X_{\Delta}\right)$. A similar computation in the case of $K$-theory is done by Anderson and Payne [2015]. Brion and Vergne [1997] (see also [Vezzosi and Vistoli 2003]) proved that the $T$-equivariant $K$-theory ring of a smooth toric variety $X_{\Delta}$ is isomorphic to the ring of integral piecewise exponential functions on the fan $\Delta$. Anderson and Payne show that for an arbitrary fan $\Delta$ this ring gives the operational $T$-equivariant $K$-cohomology of the variety $X_{\Delta}$.

One of the goals of this article is to extend Anderson and Payne's results to the case of algebraic cobordism. The $T$-equivariant algebraic cobordism of smooth toric varieties was computed by Krishna and Uma [2013]. Using the same terminology as in the case of Chow theory and $K$-theory, the equivariant cobordism ring $\Omega_{T}^{*}\left(X_{\Delta}\right)$ of a smooth quasiprojective toric variety $X_{\Delta}$ can be identified with the ring of piecewise graded power series on the fan $\Delta$, with coefficients in the Lazard ring $\mathbb{L}$. We prove below that, for any quasiprojective toric variety $X_{\Delta}$, the ring of piecewise graded power series on $\Delta$ gives the operational $T$-equivariant cobordism ring $\Omega_{T}^{*}\left(X_{\Delta}\right)$.

We start by constructing the operational bivariant theory $B^{*}$ for a suitably general class of homology theories $B_{*}$. To carry out the construction of the operational bivariant theory, it suffices to assume that $B_{*}$ is a refined oriented Borel-Moore prehomology theory (ROBM prehomology theory). This is a weakening of the notion of oriented Borel-Moore homology theory [Levine and Morel 2007, Definition 5.3.1] with refined Gysin homomorphisms, where we do not require the 
projective bundle, extended homotopy and the cellular decomposition properties. The various constructions can be summarized by a diagram as follows:

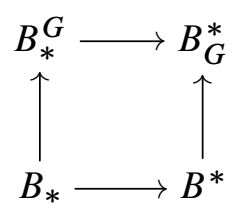

Each horizontal arrow associates to an ROBM prehomology theory its operational bivariant theory. This step can be applied to an arbitrary ROBM prehomology theory $B_{*}$, including its equivariant version $B_{*}^{G}$ for a linear algebraic group $G$. The vertical arrows associate to a theory its $G$-equivariant version using Totaro's [1999] algebraic approximation of the Borel construction from topology. For these constructions to be well-defined, we need to assume that the ROBM prehomology theory $B_{*}$ has the localization and homotopy properties. The construction of $B_{*}^{G}$ is a direct generalization of similar constructions in Chow theory by Totaro [1999] and by Edidin and Graham [1998], and in algebraic cobordism by Deshpande [2009], Krishna [2012] and Heller and Malagón-López [2013]. Note that, unlike equivariant Chow and algebraic cobordism theories, the equivariant $K$-theory is constructed not by the Borel construction, but using equivariant sheaves.

We will prove that the above square commutes; more precisely, the two ways to construct $B_{G}^{*}$ agree if we assume that the original theory $B_{*}$ has certain exact descent sequences for envelopes. Such sequences were first proved by Gillet [1984] in $K$-theory and Chow theory, and they were used by Edidin and Graham [1998] to prove the commutativity of the square above for Chow theory. The Edidin-Graham proof can be generalized to an arbitrary ROBM prehomology theory, but the descent property depends on the theory. The descent property for the algebraic cobordism theory was proved in [González and Karu 2015].

The descent property in the Chow theory was used by Kimura [1992] to give an inductive construction of operational Chow cohomology classes. We will generalize Kimura's proofs to arbitrary ROBM prehomology theories that satisfy the descent property.

Levine and Morel [2007] showed that the algebraic cobordism theory is universal among all oriented Borel-Moore homology theories. We do not know any similar universality statement for the operational cobordism theory. In any bivariant theory, multiplication with a bivariant class defines an operation on homology. This gives a functor from the bivariant theory to the operational theory constructed from its homology. Hence the operational theory is the universal target of all bivariant theories with a fixed homology theory. Yokura [2009] has proposed a geometric method for constructing a bivariant algebraic cobordism theory $\widetilde{\Omega}^{*}$, which would be universal among a class of oriented bivariant theories. The homology of this 
bivariant theory $\widetilde{\Omega}^{*}$ is expected to be the algebraic cobordism $\Omega_{*}$. By universality, there should exist a natural transformation from Yokura's bivariant $\widetilde{\Omega}^{*}$ to the operational $\Omega^{*}$, restricting to an isomorphism between the homology theories. To relate these two theories would then be an interesting problem.

We state our result describing the operational $T$-equivariant cobordism of a toric variety only in the quasiprojective case since this assumption is used in the development of equivariant cobordism in our references [Deshpande 2009; Krishna 2012; Heller and Malagón-López 2013]. However, for torus actions one could define a version of equivariant cobordism without the quasiprojectivity assumption, and our description would still hold in that setting. More precisely, the required GIT quotients for the Borel construction exist for torus actions even in the nonquasiprojective case because $T$ is a special group (see Proposition 23 in [Edidin and Graham 1998]); in addition in the case of $T$-equivariant cobordism one has induced pushforwards for proper morphisms, and then the argument provided in Section 7 goes through without the quasiprojectivity assumption.

Dependence on the refined Gysin homomorphisms for cobordism. Following Fulton and MacPherson, we associate an operational bivariant theory to any ROBM prehomology theory, which in particular must have refined Gysin homomorphisms as in Definition 2.5. Constructing the refined Gysin homomorphisms in the theory of algebraic cobordism is the most delicate part of [Levine and Morel 2007]. A reader with the case of algebraic cobordism in mind may therefore wonder how much our results depend on these homomorphisms. Refined Gysin homomorphisms appear in our definition of operational theories $B^{*}$ in Section $3 C$ (in axiom $\left(C_{3}\right)$ ), in the definition of equivariant theories $B_{*}^{G}$ in Sections $4 \mathrm{~B}-4 \mathrm{C}$ (as the maps in a directed system used to define $B_{*}^{G}$ ), in the definition of the ring structure on $B_{*}$ in the smooth case in Section 2C (as a pullback along the diagonal), in the proof of the Poincare duality isomorphism Proposition 3.2 (as a pullback along a graph morphism), and in the proof of the commutativity of the square above in Proposition 5.2 using Poincaré duality. Finally, Gysin homomorphisms appear in the theorem of Krishna and Uma, Theorem 7.2, that describes the cobordism ring of a smooth toric variety via pullback to the fixed-point set.

The paper is organized as follows. We define refined oriented Borel-Moore prehomology theories in Section 2. In Section 3 we define bivariant theories and associate the operational bivariant theory $B^{*}$ to any ROBM prehomology theory $B_{*}$ in the categories $\operatorname{Sch}_{k}$ and $G-\mathrm{Sch}_{k}$, which among other properties has the original theory $B_{*}$ as its associated homology theory (see Proposition 3.1) and has a Poincaré duality isomorphism between homology and cohomology in the nonsingular case (see Proposition 3.2). In Section 4 we start from any ROBM prehomology theory $B_{*}$ on $\operatorname{Sch}_{k}$ that satisfies the localization and homotopy properties and construct 
the $G$-equivariant ROBM prehomology theory $B_{*}^{G}$ on $G-\operatorname{Sch}_{k}$ by taking a limit over successively better approximations of the Borel construction. In Section 5 we show that if $B_{*}$ has exact descent sequences (5-1), then the computation of bivariant classes can be inductively reduced to the nonsingular case (see Theorem 5.6 and Theorem 5.3), and that furthermore the operational equivariant theory $B_{G}^{*}$ can alternatively be computed by applying the limit construction directly to the operational theory $B^{*}$ (see Proposition 5.2).

In Section 6 we overview the theory of algebraic cobordism $\Omega_{*}$. We conclude this article in Section 7 by showing in Theorem 7.3 that the operational $T$-equivariant cobordism ring of a quasiprojective toric variety can be described as the ring of piecewise graded power series on the fan with coefficients in the Lazard ring.

\section{Refined oriented Borel-Moore prehomology theories}

\section{A. Notation and conventions.}

2A.1. Throughout this article all of our schemes will be defined over a fixed field $k$. We denote by $\operatorname{Sch}_{k}$ the category of separated finite-type schemes over Spec $k$ and by $\mathrm{Sch}_{k}^{\prime}$ the subcategory of $\mathrm{Sch}_{k}$ with the same objects but whose morphisms are the projective morphisms. We denote by $\mathrm{Sm}_{k}$ the full subcategory of $\mathrm{Sch}_{k}$ of smooth and quasiprojective schemes. By a smooth morphism we always mean a smooth and quasiprojective morphism. Ab $b_{*}$ will denote the category of graded abelian groups.

2A.2. Let $G$ be a linear algebraic group. A $G$-linearization of a line bundle $f: L \rightarrow X$ over the $G$-scheme $X$ is a $G$-action $\Phi: G \times L \rightarrow L$ on $L$ such that $f$ is $G$-equivariant and for every $x \in X$ and $g \in G$ the action map $\Phi_{g}: L_{x} \rightarrow L_{g x}$ is linear. We denote by $G-S_{c h}$ the category whose objects are the separated finitetype $G$-schemes over Spec $k$ that admit an ample $G$-linearizable line bundle and whose morphisms are $G$-equivariant morphisms. We denote by $G-\mathrm{Sch}_{k}^{\prime}$ the subcategory of $G-\mathrm{Sch}_{k}$ with the same objects but whose morphisms are the projective $G$-equivariant morphisms. Note that all schemes in $G-\mathrm{Sch}_{k}$ are assumed to be quasiprojective; this is needed in the construction of equivariant theories using the GIT quotients.

2A.3. In Sections 5-7 we will assume that $k$ has characteristic zero and in Sections 4B-4D we will assume that $k$ is infinite. The assumption on the characteristic of $k$ is only meant to guarantee the existence of smooth projective envelopes in the categories $\operatorname{Sch}_{k}$ and $G-\mathrm{Sch}_{k}$ and to provide the setting for the use of the Levine-Pandharipande version of algebraic cobordism, which requires resolution of singularities by projective morphisms, weak factorization for birational maps and some Bertini-type theorems that hold in characteristic zero. The assumption on the cardinality of $k$ is only used explicitly in the proof of Proposition 4.3. 
2A.4. We call a morphism $f: Z \rightarrow X$ in one of the categories $\mathscr{C}=\operatorname{Sch}_{k}$ or $\mathscr{C}=G-\mathrm{Sch}_{k}$ a locally complete intersection morphism in $\mathscr{C}$, or simply an lci morphism in $\mathscr{C}$, if there exist a regular embedding $i: Z \rightarrow Y$ and a smooth morphism $g: Y \rightarrow X$, with $g$ and $i$ in $\mathscr{C}$ such that $f=g i$. When we work in the category $G-\operatorname{Sch}_{k}$ and we say that a morphism $f$ is an equivariant lci morphism, or simply an lci morphism, we mean that $f$ is an lci morphism in $G-\mathrm{Sch}_{k}$. We follow the convention that smooth morphisms, and more generally lci morphisms, are assumed to have a relative dimension. If $f: X \rightarrow Y$ is an lci morphism of relative dimension $d$ (or relative codimension $-d$ ) and $Y$ is irreducible, then $X$ is a scheme of pure dimension equal to $\operatorname{dim} Y+d$.

\section{B. ROBM prehomology theories.}

2B.1. For simplicity, we unify the treatment of the cases when the ambient category is $\operatorname{Sch}_{k}$ or $G-\mathrm{Sch}_{k}$ for some algebraic group $G$. Therefore, through the rest of this section we fix the category $\mathscr{C}$, which is either $\operatorname{Sch}_{k}$ or $G-\operatorname{Sch}_{k}$, and we assume that all the schemes and morphisms are in $\mathscr{C}$ (e.g., the statement for any morphism should be interpreted as for any morphism in $\mathscr{C}$ ). Likewise, when $\mathscr{C}=G-\mathrm{Sch}_{k}$, by an lci morphism we mean an equivariant lci morphism. The category $\mathscr{C}^{\prime}$ is defined to be $\operatorname{Sch}_{k}^{\prime}$ or $G-\operatorname{Sch}_{k}^{\prime}$, depending on whether $\mathscr{b}$ is equal to $\operatorname{Sch}_{k}$ or $G-\operatorname{Sch}_{k}$, respectively.

Let us start by recalling the definition of a Borel-Moore functor on $\mathscr{C}$, and several extra structures on it, from [Levine and Morel 2007].

Definition 2.1. A Borel-Moore functor on $\mathscr{C}$ is given by:

- $\left(\mathrm{D}_{1}\right)$ An additive functor $H_{*}: \mathscr{C}^{\prime} \rightarrow \mathrm{Ab}_{*}$, i.e., a functor $H_{*}: \mathscr{C}^{\prime} \rightarrow \mathrm{Ab} b_{*}$ such that, for any finite family $\left(X_{1}, \ldots, X_{r}\right)$ of schemes in $\mathscr{C}^{\prime}$, the morphism

$$
\bigoplus_{i=1}^{r} H_{*}\left(X_{i}\right) \rightarrow H_{*}\left(\coprod_{i=1}^{r} X_{i}\right)
$$

induced by the projective morphisms $X_{i} \subseteq \coprod_{i=1}^{r} X_{i}$ is an isomorphism.

- $\left(\mathrm{D}_{2}\right)$ For each smooth equidimensional morphism $f: Y \rightarrow X$ of relative dimension $d$ in $\mathscr{C}$, a homomorphism of graded groups

$$
f^{*}: H_{*}(X) \rightarrow H_{*+d}(Y) .
$$

These data satisfy the following axioms:

- $\left(\mathrm{A}_{1}\right)$ For any pair of composable smooth equidimensional morphisms ( $f: Y \rightarrow X$, $g: Z \rightarrow Y$ ) of relative dimensions $d$ and $e$ respectively, one has

$$
(f \circ g)^{*}=g^{*} \circ f^{*}: H_{*}(X) \rightarrow H_{*+d+e}(Z) .
$$

In addition, $\operatorname{Id}_{X}^{*}=\operatorname{Id}_{H_{*}(X)}$ for any $X \in \mathscr{C}$. 
- $\left(\mathrm{A}_{2}\right)$ For any projective morphism $f: X \rightarrow Z$ and any smooth equidimensional morphism $g: Y \rightarrow Z$, if one forms the fiber diagram

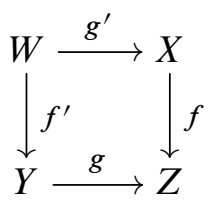

then

$$
g^{*} f_{*}=f_{*}^{\prime} g^{\prime *} .
$$

Notation 2.2. For each projective morphism $f$ the homomorphism $H_{*}(f)$ is denoted by $f_{*}$ and is called the pushforward along $f$. For each smooth equidimensional morphism $g$ the homomorphism $g^{*}$ is called the pullback along $g$.

Definition 2.3. A Borel-Moore functor with exterior product on $\mathscr{b}$ consists of a Borel-Moore functor $H_{*}$ on $\mathscr{C}$, together with:

- $\left(\mathrm{D}_{3}\right)$ An element $1 \in H_{0}(\operatorname{Spec} k)$, and for each pair $(X, Y)$ of schemes in $\mathscr{C}$ a bilinear graded pairing (called the exterior product)

$$
\begin{aligned}
\times: H_{*}(X) \times H_{*}(Y) & \rightarrow H_{*}(X \times Y), \\
(\alpha, \beta) & \mapsto \alpha \times \beta
\end{aligned}
$$

which is (strictly) commutative, associative, and admits 1 as unit.

These satisfy:

- $\left(\mathrm{A}_{3}\right)$ Given projective morphisms $f: X \rightarrow X^{\prime}$ and $g: Y \rightarrow Y^{\prime}$, one has that for any classes $\alpha \in H_{*}(X)$ and $\beta \in H_{*}(Y)$

$$
(f \times g)_{*}(\alpha \times \beta)=f_{*}(\alpha) \times g_{*}(\beta) \in H_{*}\left(X^{\prime} \times Y^{\prime}\right) .
$$

- $\left(\mathrm{A}_{4}\right)$ Given smooth equidimensional morphisms $f: X \rightarrow X^{\prime}$ and $g: Y \rightarrow Y^{\prime}$, one has that for any classes $\alpha \in H_{*}\left(X^{\prime}\right)$ and $\beta \in H_{*}\left(Y^{\prime}\right)$

$$
(f \times g)^{*}(\alpha \times \beta)=f^{*}(\alpha) \times g^{*}(\beta) \in H_{*}(X \times Y) .
$$

Remark 2.4. Given a Borel-Moore functor with exterior product $H_{*}$, the axioms give $H_{*}(\operatorname{Spec} k)$ a commutative, graded ring structure, give to each $H_{*}(X)$ the structure of $H_{*}(\operatorname{Spec} k)$-module, and imply that the operations $f_{*}$ and $f^{*}$ preserve the $H_{*}(\operatorname{Spec} k)$-module structure.

Definition 2.5. A Borel-Moore functor with intersection products on $\mathscr{b}$ is a BorelMoore functor $H_{*}$ on $\mathscr{C}$, together with: 
- $\left(\mathrm{D}_{4}\right)$ For each lci morphism $f: Z \rightarrow X$ of relative codimension $d$ and any morphism $g: Y \rightarrow X$ giving the fiber diagram

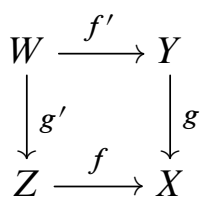

a homomorphism of graded groups

$$
f_{g}^{!}: H_{*}(Y) \rightarrow H_{*-d}(W)
$$

These satisfy:

- (A) If $f_{1}: Z_{1} \rightarrow X$ and $f_{2}: Z_{2} \rightarrow Z_{1}$ are lci morphisms and $g: Y \rightarrow X$ any morphism giving the fiber diagram

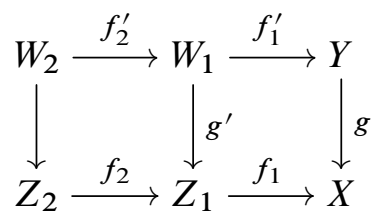

one has $\left(f_{1} \circ f_{2}\right)_{g}^{!}=\left(f_{2}\right)_{g^{\prime}} \circ\left(f_{1}\right)_{g}^{!}$.

- (A) If $f_{1}: Z_{1} \rightarrow X_{1}$ and $f_{2}: Z_{2} \rightarrow X_{2}$ are lci morphisms of relative codimensions $d$ and $e$, respectively, and $h_{1}: Y \rightarrow X_{1}$ and $h_{2}: Y \rightarrow X_{2}$ are arbitrary morphisms giving the fiber diagram

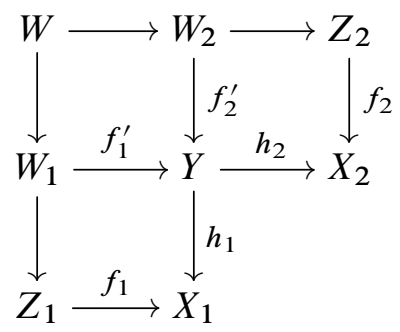

one has $\left(f_{1}\right) !_{h_{1} f_{2}^{\prime}} \circ\left(f_{2}\right) !_{h_{2}}^{!}=\left(f_{2}\right)_{h_{2} f_{1}^{\prime}} \circ\left(f_{1}\right)_{h_{1}}^{!}: B_{*} Y \rightarrow B_{*-d-e} W$.

- $\left(\mathrm{A}_{7}\right)$ For any smooth morphism $f: Y \rightarrow X$ one has $f_{\mathrm{Id}_{X}}^{!}=f^{*}$. 
For any lci morphism $f: Z \rightarrow X$, any morphism $g: Y \rightarrow X$ and any morphism $h: Y^{\prime} \rightarrow Y$, if one forms the fiber diagram

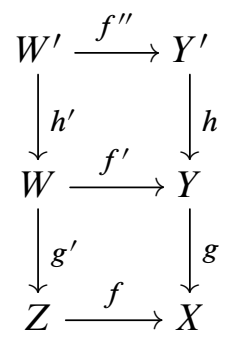

then:

- $\left(\mathrm{A}_{8}\right)$ If $g$ and $f$ are Tor-independent in $\operatorname{Sch}_{k}$ (i.e., if $\operatorname{Tor}_{j}^{O_{X}}\left(\mathrm{O}_{Y}, \mathrm{O}_{Z}\right)=0$ for all $j>0)$ then

$$
f_{g h}^{!}=\left(f^{\prime}\right)_{h}^{!}
$$

- (A9) If $h$ is projective then $f_{g}^{!} \circ h_{*}=h_{*}^{\prime} \circ f_{g h}^{!}$.

- $\left(\mathrm{A}_{10}\right)$ If $h$ is smooth equidimensional then $f_{g h}^{!} \circ h^{*}=h^{*} \circ f_{g}^{!}$.

Notation 2.6. Given a Borel-Moore functor with intersection products $H_{*}$, for any lci morphism $f: Z \rightarrow X$ of relative codimension $d$, the map $f_{\mathrm{Id}_{X}}^{!}: H_{*}(X) \rightarrow$ $H_{*-d}(Z)$ is called the lci pullback along $f$ and denoted by $f^{*}$. For each lci morphism $f: Z \rightarrow X$ and each morphism $g: Y \rightarrow X$ we call the morphism $f_{g}^{!}$the refined lci pullback along $f$ associated to $g$. We will usually denote $f_{g}^{!}$ simply by $f^{!}$with an indication of where it acts. When the lci morphism $f$ is a regular embedding then $f_{g}^{!}$is called a refined Gysin homomorphism. Refined Gysin homomorphisms and smooth pullbacks can be composed to construct all refined lci pullbacks.

Definition 2.7. A Borel-Moore functor with compatible exterior and intersection products on $\mathscr{C}$ consists of a Borel-Moore functor $H_{*}$ on $\mathscr{C}$ endowed with exterior products and intersection products that in addition satisfy:

- ( $\left.\mathrm{A}_{11}\right)$ If, for $i=1$ and $i=2, f_{i}: Z_{i} \rightarrow X_{i}$ is an lci morphism and $g_{i}: Y_{i} \rightarrow X_{i}$ is an arbitrary morphism, and one forms the fiber diagram

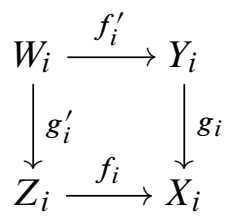

one has that for any classes $\alpha_{1} \in H_{*}\left(Y_{1}\right)$ and $\alpha_{2} \in H_{*}\left(Y_{2}\right)$

$$
\left(f_{1} \times f_{2}\right)_{g_{1} \times g_{2}}^{!}\left(\alpha_{1} \times \alpha_{2}\right)=\left(f_{1}\right)_{g_{1}}^{!}\left(\alpha_{1}\right) \times\left(f_{2}\right)_{g_{2}}^{!}\left(\alpha_{2}\right) \in H_{*}\left(W_{1} \times W_{2}\right) \text {. }
$$


Notation 2.8. We will call a Borel-Moore functor with compatible exterior and intersection products a refined oriented Borel-Moore prehomology theory (ROBM prehomology theory, for short).

Examples of ROBM prehomology theories are Chow theory $A_{*}$ (see [Fulton 1998]), $K$-theory (i.e., the Grothendieck $K$-group functor $\mathrm{G}_{0}$ of the category of coherent $\mathrm{O}_{X}$-modules, graded by $\mathrm{G}_{0} \otimes_{\mathbb{Z}} \mathbb{Z}\left[\beta, \beta^{-1}\right]$ — see [Levine and Morel 2007, Example 2.2.5]), and algebraic cobordism $\Omega_{*}$ (see [Levine and Morel 2007]) on the category $\operatorname{Sch}_{k}$; and equivariant Chow theory $A_{*}^{G}$ and equivariant algebraic cobordism $\Omega_{*}^{G}$ on the category $G-\operatorname{Sch}_{k}$ constructed as in Section 4 (see [Edidin and Graham 1998; Krishna 2012; Heller and Malagón-López 2013]).

Levine and Morel [2007] considered the notion of an oriented Borel-Moore homology theory, which is an ROBM prehomology theory but with lci pullbacks only instead of refined lci pullbacks, and with additional axioms called projective bundle, extended homotopy and cellular decomposition properties. Because of the refined lci pullbacks, an oriented Borel-Moore homology theory is not necessarily an ROBM prehomology theory. However, one can construct refined lci pullbacks from ordinary lci pullbacks by the deformation to the normal cone argument of Fulton and MacPherson, provided that the theory additionally satisfies the homotopy and localization properties (see Section 4 for these properties). We will need the homotopy and localization properties when working with equivariant theories; hence an alternative theory that is sufficient for the constructions below would be a Borel-Moore functor with compatible lci pullbacks and exterior products, which additionally satisfies the homotopy and localization properties.

Definition 2.9. If $H_{*}$ is an ROBM prehomology theory, for any line bundle $L \rightarrow Y$ in $\mathscr{C}$ with zero section $s: Y \rightarrow L$ one defines the operator $\tilde{\mathrm{c}}_{1}(L): H_{*}(Y) \rightarrow H_{*-1}(Y)$ by $\tilde{\mathrm{c}}_{1}(L)=s^{*} s_{*}$, and calls it the first Chern class operator of $L$.

2C. Cohomology theory. Let $H_{*}$ be an ROBM prehomology theory. For a smooth scheme $X$ of pure dimension $n$, define

$$
H^{*}(X)=H_{n-*}(X)
$$

For an arbitrary smooth scheme we extend this notion by taking the direct sum over pure-dimensional parts of $X$.

The groups $H^{*}(X)$ are commutative graded rings with unit, with product defined by

$$
\begin{aligned}
H^{*}(X) \times H^{*}(X) & \rightarrow H^{*}(X), \\
(a, b) & \mapsto \Delta_{X}^{*}(a \times b),
\end{aligned}
$$


where $\Delta_{X}: X \rightarrow X \times X$ is the diagonal map and $\Delta_{X}^{*}$ is the lci pullback. Associativity of the product follows from $\left(\mathrm{A}_{5}\right)$ and $\left(\mathrm{A}_{11}\right)$ applied to two different ways to construct the diagonal $X \rightarrow X \times X \times X$ by composing $\Delta_{X}$.

Let $\pi: X \rightarrow$ Spec $k$ be the structure morphism, and define $1_{X}=\pi^{*}(1) \in H^{0}(X)$, where $1=1_{\text {Spec } k} \in H^{0}(\operatorname{Spec} k)$ is the element specified in $\left(D_{3}\right)$. Then $1_{X}$ is the multiplicative identity in the ring $H^{*}(X)$.

Axioms $\left(\mathrm{A}_{5}\right)$ and $\left(\mathrm{A}_{11}\right)$ imply that, if $f: X \rightarrow Y$ is an lci morphism between smooth schemes, then $f^{*}: H^{*}(Y) \rightarrow H^{*}(X)$ is a homomorphism of graded rings with unit. Thus, we may view $H^{*}$ as a contravariant functor from the category of smooth schemes and lci morphisms in $\mathscr{b}$ to the category of commutative graded rings with unit. In the next section we extend this functor to the whole category $\mathscr{C}$.

\section{Operational bivariant theories}

In this section we consider a refined oriented Borel-Moore prehomology theory $B_{*}$ on one of the categories $\mathscr{C}=\operatorname{Sch}_{k}$ or $\mathscr{C}=G-\operatorname{Sch}_{k}$, and associate to it a bivariant theory $B^{*}$ on $\mathscr{C}$. We present a unified treatment of these two cases. Therefore throughout this section we fix one of these two categories and denote it by $\mathscr{C}$, and we assume that all the schemes and morphisms are in $\mathscr{b}$ following the conventions described in Section 2B.1.

Operational theories were defined for general homology theories by Fulton and MacPherson [1981]. The constructions that we present in this section follow [Fulton 1998, Chapter 17] where the operational theory $A^{*}$ is constructed for the Chow theory $A_{*}$. Some definitions and proofs have been modified to adapt them to our setting.

3A. Bivariant theories. A bivariant theory $B^{*}$ on $\mathscr{C}$ assigns to each morphism $f: X \rightarrow Y$ in $\mathscr{C}$ a graded abelian group $B^{*}(X \rightarrow Y)$. The groups $B^{*}(X \rightarrow Y)$ are endowed with three operations called product, pushforward and pullback, which are mutually compatible and admit units:

- $\left(\mathrm{P}_{1}\right)$ Product. For all morphisms $f: X \rightarrow Y$ and $g: Y \rightarrow Z$, and all integers $p$ and $q$, there is a homomorphism

$$
B^{p}(X \stackrel{f}{\longrightarrow} Y) \otimes B^{q}(Y \stackrel{g}{\longrightarrow} Z) \stackrel{\cdot}{\longrightarrow} B^{p+q}(X \stackrel{g f}{\longrightarrow} Z) .
$$

The image of $c \otimes d$ is denoted $c \cdot d$.

- $\left(\mathrm{P}_{2}\right)$ Pushforward. If $f: X \rightarrow Y$ is a projective morphism, $g: Y \rightarrow Z$ is any morphism and $p$ is an integer, there is a homomorphism

$$
f_{*}: B^{p}(X \stackrel{g f}{\longrightarrow} Z) \rightarrow B^{p}(Y \stackrel{g}{\longrightarrow} Z) .
$$


- $\left(\mathrm{P}_{3}\right)$ Pullback. If $f: X \rightarrow Y$ and $g: Y^{\prime} \rightarrow Y$ are arbitrary morphisms, $f^{\prime}: X^{\prime}=$ $X \times{ }_{Y} Y^{\prime} \rightarrow Y^{\prime}$ is the projection and $p$ is an integer, there is a homomorphism

$$
g^{*}: B^{p}(X \stackrel{f}{\longrightarrow} Y) \rightarrow B^{p}\left(X^{\prime} \stackrel{f^{\prime}}{\longrightarrow} Y^{\prime}\right) .
$$

- (U) Units. For each $X$ there is an element $1_{X} \in B^{0}(X \stackrel{\text { Id }}{\longrightarrow} X)$ such that $\alpha \cdot 1_{X}=\alpha$ and $1_{X} \cdot \beta=\beta$ for all morphisms $W \rightarrow X$ and $X \rightarrow Y$ and all classes $\alpha \in B^{*}(W \rightarrow X)$ and $\beta \in B^{*}(X \rightarrow Y)$. These unit elements are compatible with pullbacks, i.e., $g^{*}\left(1_{X}\right)=1_{Z}$ for all morphisms $g: Z \rightarrow X$.

These operations are required to satisfy seven compatibility properties:

- $\left(\mathrm{B}_{1}\right)$ Associativity of products. If $c \in B^{*}(X \rightarrow Y), d \in B^{*}(Y \rightarrow Z)$ and $e \in B^{*}(Z \rightarrow W)$, then

$$
(c \cdot d) \cdot e=c \cdot(d \cdot e) \in B^{*}(X \rightarrow W) .
$$

- $\left(\mathrm{B}_{2}\right)$ Functoriality of pushforwards. If $c \in B^{*}(X \rightarrow Y)$, then $\operatorname{Id}_{X *} c=c \in$ $B^{*}(X \rightarrow Y)$. Moreover, if $f: X \rightarrow Y$ and $g: Y \rightarrow Z$ are projective morphisms, $Z \rightarrow W$ is arbitrary, and $d \in B^{*}(X \rightarrow W)$, then

$$
(g f)_{*} d=g_{*}\left(f_{*} d\right) \in B^{*}(Z \rightarrow W) .
$$

- $\left(\mathrm{B}_{3}\right)$ Functoriality of pullbacks. If $c \in B^{*}(X \rightarrow Y)$, then $\operatorname{Id}_{Y}^{*} c=c \in B^{*}(X \rightarrow Y)$. Moreover, if $f: X \rightarrow Y, g: Y^{\prime} \rightarrow Y$ and $h: Y^{\prime \prime} \rightarrow Y^{\prime}$ are arbitrary morphisms, $X^{\prime \prime}=X \times_{Y} Y^{\prime \prime} \rightarrow Y^{\prime \prime}$ is the projection, and $d \in B^{*}(X \rightarrow Y)$, then

$$
(g h)^{*} d=h^{*}\left(g^{*} d\right) \in B^{*}\left(X^{\prime \prime} \rightarrow Y^{\prime \prime}\right) .
$$

- $\left(\mathrm{B}_{12}\right)$ Product and pushforward commute. If $f: X \rightarrow Y$ is projective, $Y \rightarrow Z$ and $Z \rightarrow W$ are arbitrary, and $c \in B^{*}(X \rightarrow Z)$ and $d \in B^{*}(Z \rightarrow W)$, then

$$
f_{*}(c) \cdot d=f_{*}(c \cdot d) \in B^{*}(Y \rightarrow W) .
$$

- $\left(\mathrm{B}_{13}\right)$ Product and pullback commute. If $c \in B^{*}(X \stackrel{f}{\longrightarrow} Y)$ and $d \in B^{*}(Y \stackrel{g}{\longrightarrow} Z)$, and $h: Z^{\prime} \rightarrow Z$ is arbitrary, and one forms the fiber diagram

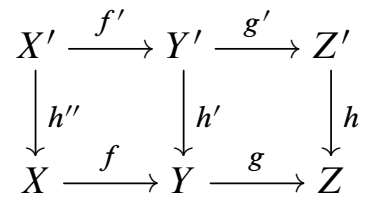

then

$$
(h)^{*}(c \cdot d)=h^{* *}(c) \cdot h^{*}(d) \in B^{*}\left(X^{\prime} \rightarrow Z^{\prime}\right) .
$$


- $\left(\mathrm{B}_{23}\right)$ Pushforward and pullback commute. If $f: X \rightarrow Y$ is projective, $g: Y \rightarrow Z$ and $h: Z^{\prime} \rightarrow Z$ are arbitrary morphisms, and $c \in B^{*}(X \rightarrow Z)$, and one forms the fiber diagram

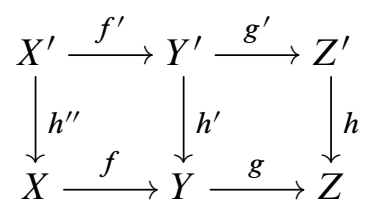

then

$$
h^{*}\left(f_{*} c\right)=f_{*}^{\prime}\left(h^{*} c\right) \in B^{*}\left(Y^{\prime} \rightarrow Z^{\prime}\right) .
$$

- $\left(\mathrm{B}_{123}\right)$ Projection formula. If $f: X \rightarrow Y$ and $g: Y \rightarrow Z$ are arbitrary morphisms, $h^{\prime}: Y^{\prime} \rightarrow Y$ is projective and $c \in B^{*}(X \rightarrow Y)$ and $d \in B^{*}\left(Y^{\prime} \rightarrow Z\right)$, and one forms the fiber diagram

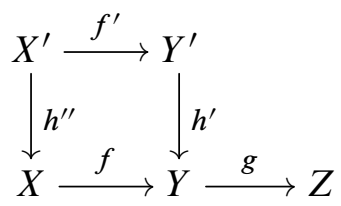

then

$$
c \cdot h_{*}^{\prime}(d)=h_{*}^{\prime \prime}\left(h^{\prime *}(c) \cdot d\right) \in B^{*}(X \rightarrow Z) .
$$

The group $B^{p}(X \stackrel{f}{\longrightarrow} Y)$ may be denoted by simply by $B^{p}(X \rightarrow Y)$ or $B^{p}(f)$. We will denote by $B^{*}(X \longrightarrow Y), B^{*}(X \rightarrow Y)$ or $B^{*}(f)$ the direct sum of all $B^{p}(X \underset{f}{\longrightarrow} Y)$, for $p \in \mathbb{Z}$.

3B. Homology and cohomology. A bivariant theory $B^{*}(X \rightarrow Y)$ contains both a covariant homology theory $B_{*}(X)$ and a contravariant cohomology theory $B^{*}(X)$.

The homology is defined by $B_{p}(X)=B^{-p}(X \rightarrow$ Spec $k)$. For any projective morphism $f: X \rightarrow Y$, the pushforward in the bivariant theory defines the functorial pushforward map in homology $f_{*}: B_{*}(X) \rightarrow B_{*}(Y)$.

The cohomology is defined by $B^{p}(X)=B^{p}\left(\operatorname{Id}_{X}: X \rightarrow X\right)$. The product operation in the bivariant theory turns $B^{*}(X)$ into a graded ring with unit $1_{X}$ and turns $B_{*}(X)$ into a graded left module over $B^{*}(X)$. The product operation $B^{*}(X) \times B_{*}(X) \rightarrow B_{*}(X)$ is called the cap product and denoted $(\alpha, \beta) \mapsto \alpha \cap \beta$. For any morphism $f: X \rightarrow Y$, the pullback in the bivariant theory defines a functorial pullback $f^{*}: B^{*}(Y) \rightarrow B^{*}(X)$. The pullback map is a homomorphism of graded rings. When $f$ is a projective morphism, the projection formula relates the pullback, pushforward, and cap product as follows:

$$
f_{*}\left(f^{*}(\alpha) \cap \beta\right)=\alpha \cap f_{*}(\beta) .
$$


3C. Operational bivariant theories. We now fix an ROBM prehomology theory $B_{*}$ on $\mathscr{C}$, and associate a bivariant theory $B^{*}$ to it.

Let $f: X \rightarrow Y$ be any morphism. For each morphism $g: Y^{\prime} \rightarrow Y$, form the fiber square

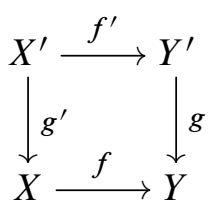

with induced morphisms as labeled. An element $c$ in $B^{p}(X \stackrel{f}{\longrightarrow} Y)$, called a bivariant class, is a collection of homomorphisms

$$
c_{g}^{(m)}: B_{m} Y^{\prime} \rightarrow B_{m-p} X^{\prime}
$$

for all $g: Y^{\prime} \rightarrow Y$ and all $m \in \mathbb{Z}$, compatible with projective pushforwards, smooth pullbacks, intersection products and exterior products, that is:

- $\left(\mathrm{C}_{1}\right)$ If $h: Y^{\prime \prime} \rightarrow Y^{\prime}$ is projective and $g: Y^{\prime} \rightarrow Y$ is arbitrary, and one forms the fiber diagram

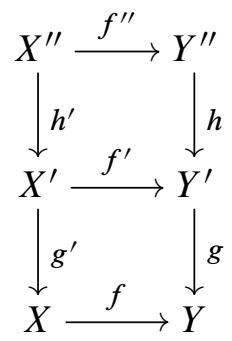

then, for all $\alpha \in B_{m}\left(Y^{\prime \prime}\right)$,

$$
c_{g}^{(m)}\left(h_{*} \alpha\right)=h_{*}^{\prime} c_{g h}^{(m)}(\alpha)
$$

in $B_{m-p}\left(X^{\prime}\right)$.

- $\left(\mathrm{C}_{2}\right)$ If $h: Y^{\prime \prime} \rightarrow Y^{\prime}$ is smooth of relative dimension $n$ and $g: Y^{\prime} \rightarrow Y$ is arbitrary, and one forms the fiber diagram

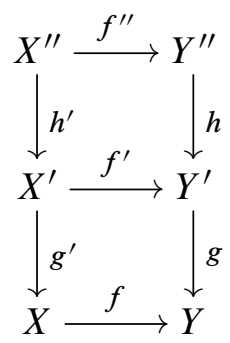


then, for all $\alpha \in B_{m}\left(Y^{\prime}\right)$,

$$
c_{g h}^{(m+n)}\left(h^{*} \alpha\right)=h^{\prime *} c_{g}^{(m)}(\alpha)
$$

in $B_{m+n-p}\left(X^{\prime \prime}\right)$.

- $\left(\mathrm{C}_{3}\right)$ If $g: Y^{\prime} \rightarrow Y$ and $h: Y^{\prime} \rightarrow Z^{\prime}$ are morphisms, and $i: Z^{\prime \prime} \rightarrow Z^{\prime}$ is an lci morphism of codimension $e$, and one forms the fiber diagram

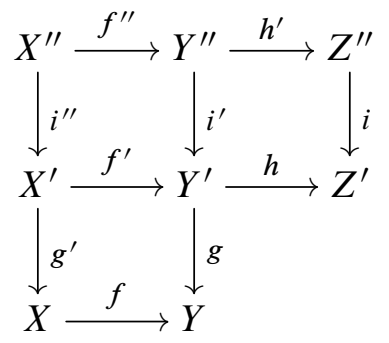

then for all $\alpha \in B_{m}\left(Y^{\prime}\right)$,

$$
c_{g i^{\prime}}^{(m-e)}\left(i !^{!} \alpha\right)=i^{!} c_{g}^{(m)}(\alpha)
$$

in $B_{m-e-p}\left(X^{\prime \prime}\right)$.

- $\left(\mathrm{C}_{4}\right)$ If $g: Y^{\prime} \rightarrow Y$ is arbitrary, and $h: Y^{\prime} \times Z \rightarrow Y^{\prime}$ and $h^{\prime}: X^{\prime} \times Z \rightarrow X^{\prime}$ are the projections, and one forms the fiber diagram

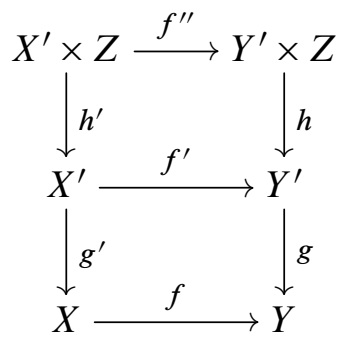

then, for all $\alpha \in B_{m}\left(Y^{\prime}\right)$ and $\beta \in B_{l}(Z)$,

$$
c_{g h}^{(m+l)}(\alpha \times \beta)=c_{g}^{(m)}(\alpha) \times \beta
$$

in $B_{m+l-p}\left(X^{\prime} \times Z\right)$.

The three operations are defined as follows: 
- Product: Let $c \in B^{p}(X \stackrel{f}{\longrightarrow} Y)$ and $d \in B^{q}(Y \stackrel{g}{\longrightarrow} Z)$. Given any morphism $h: Z^{\prime} \rightarrow Z$, form the fiber diagram

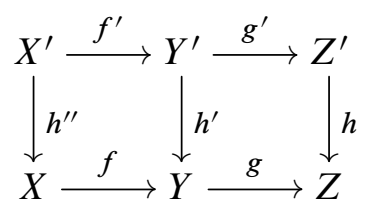

and for each integer $m$ define $(c \cdot d)_{h}^{(m)}=c_{h^{\prime}}^{(m-q)} \circ d_{h}^{(m)}: B_{m} Z^{\prime} \rightarrow B_{m-p-q} X^{\prime}$.

- Pushforward: Given $c \in B^{p}(X \stackrel{f}{\longrightarrow} Y \stackrel{g}{\longrightarrow} Z)$ and any morphism $h: Z^{\prime} \rightarrow Z$, form the fiber diagram

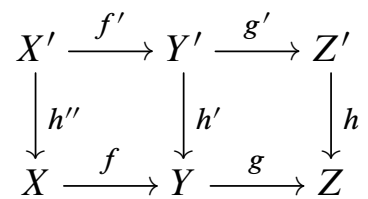

and for each integer $m$ define $\left(f_{*} c\right)_{h}^{(m)}=f_{*}^{\prime} \circ c_{h}^{(m)}: B_{m} Z^{\prime} \rightarrow B_{m-p} Y^{\prime}$.

- Pullback: Given $c \in B^{p}(X \stackrel{f}{\longrightarrow} Y)$ and morphisms $g: Y^{\prime} \rightarrow Y$ and $h: Y^{\prime \prime} \rightarrow Y^{\prime}$, form the fiber diagram

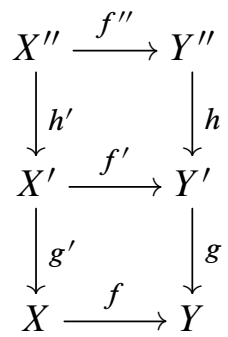

and for each integer $m$ define $\left(g^{*} c\right)_{h}^{(m)}=c_{g h}^{(m)}: B_{m} Y^{\prime \prime} \rightarrow B_{m-p} X^{\prime \prime}$.

It is straightforward to verify that these three operations are well-defined (i.e., that $c \cdot d, f_{*} c$ and $g^{*} c$ satisfy $\left(\mathrm{C}_{1}\right)-\left(\mathrm{C}_{4}\right)$, so that they define classes in the appropriate bivariant groups). For each $X$, unit elements $1_{X} \in B^{0}(X \rightarrow X)$ satisfying the property $(\mathrm{U})$ are defined by letting them act by identity homomorphisms. It is also straightforward to check that the three operations satisfy properties $\left(B_{1}\right)-\left(B_{123}\right)$. In conclusion, the operational theory is a bivariant theory.

The only bivariant theories we will consider are the operational ones. By a bivariant theory we will mean an operational bivariant theory associated to an ROBM prehomology theory. 
3D. Homology and cohomology for operational bivariant theories. Recall that any bivariant theory $B^{*}(X \rightarrow Y)$ contains both a covariant homology theory $B^{-*}(X \rightarrow$ Spec $k)$ and a contravariant cohomology theory $B^{*}\left(\operatorname{Id}_{X}: X \rightarrow X\right)$. We claim that if the bivariant theory $B^{*}(X \rightarrow Y)$ is the operational theory associated to an ROBM prehomology theory $B_{*}$, then the homology theory $B^{-*}(X \rightarrow \operatorname{Spec} k)$ is isomorphic to the original theory $B_{*}(X)$. Similarly, the cohomology theory $B^{*}(X \rightarrow X)$ agrees with the cohomology theory $B^{*}(X)$ constructed in Section 2C for smooth schemes $X$. The proofs in this section are adapted from the proofs in [Fulton 1998] for the Chow theory.

Proposition 3.1. For any $X$ and each integer $p$, the homomorphism

$$
\varphi: B^{-p}(X \rightarrow \operatorname{Spec} k) \rightarrow B_{p}(X)
$$

taking a bivariant class $c$ to $c(1)$ is an isomorphism. Here $1=1_{\operatorname{Spec} k} \in B_{0}(\operatorname{Spec} k)$ is the element specified by $\left(\mathrm{D}_{3}\right)$ in Definition 2.3. The isomorphism $\varphi$ is natural with respect to pushforwards along projective morphisms.

Proof. Define a homomorphism $\psi: B_{p}(X) \rightarrow B^{-p}(X \rightarrow$ Spec $k)$ as follows: given any $a \in B_{p}(X)$, any morphism $f: Y \rightarrow$ Spec $k$ and a class $\alpha \in B_{m}(Y)$, we set $\psi(a)(\alpha)=a \times \alpha \in B_{m+p}(X \times Y)$. It follows at once that $\psi(a)$ satisfies $\left(\mathrm{C}_{1}\right)-\left(\mathrm{C}_{4}\right)$, and $\psi$ is a well-defined homomorphism.

For each $a \in B_{p}(X)$, one has $\varphi(\psi(a))=\psi(a)(1)=a \times 1=a \in B_{p}(X)$, so $\varphi \circ \psi$ is the identity. Given any $c \in B^{-p}(X \rightarrow \operatorname{Spec} k)$, any morphism $Y \rightarrow \operatorname{Spec} k$ and any class $\alpha \in B_{m}(Y)$, one has $\psi(\varphi(c))(\alpha)=\psi(c(1))(\alpha)=c(1) \times \alpha=c(1 \times \alpha)=$ $c(\alpha) \in B^{m+p}(X \times Y)$, so $\psi \circ \varphi$ is also the identity.

Naturality of $\varphi$ with respect to projective pushforwards follows from the definition of pushforward in the operational bivariant theory.

Let us now consider the cohomology theory $B^{*}(X \rightarrow X)$. Note that an element $c \in B^{p}(X \rightarrow X)$ is a collection of homomorphisms $c_{f}^{(m)}: B_{m} X^{\prime} \rightarrow B_{m-p} X^{\prime}$, for all morphisms $f: X^{\prime} \rightarrow X$ and all integers $m$, that are compatible with projective pushforwards, smooth pullbacks, and exterior and intersection products. Using the previous proposition, we identify $B_{*}(X)$ with $B^{-*}(X \rightarrow$ Spec $k)$, and thus give $B_{*}(X)$ the structure of a module over $B^{*}(X \rightarrow X)$.

Proposition 3.2 (Poincaré duality). Let $X$ be a smooth purely $n$-dimensional scheme and let $B^{*}(X)=B_{n-*}(X)$ be the cohomology theory defined in Section $2 C$. The homomorphism defined by cap product with $1_{X} \in B^{0}(X)$,

$$
\varphi: B^{*}\left(X \stackrel{\mathrm{Id}_{X}}{\longrightarrow} X\right) \stackrel{\cap 1_{X}}{\longrightarrow} B^{*}(X),
$$

is an isomorphism of graded rings that takes $1_{X} \in B^{0}(X \rightarrow X)$ to $1_{X} \in B^{0}(X)$. The isomorphism $\varphi$ is natural with respect to pullbacks by lci morphisms. 
Proof. Let us fix an integer $p$ and define a homomorphism $\psi: B^{p}(X)=B_{n-p}(X) \rightarrow$ $B^{p}(X \rightarrow X)$ as follows: given any $a \in B_{n-p}(X)$, any morphism $f: Y \rightarrow X$ and a class $\alpha \in B_{m}(Y)$, we set $\psi(a)(\alpha)=\psi(a)_{f}^{(m)}(\alpha)=\gamma_{f}^{*}(a \times \alpha) \in B_{m-p} Y$, where $\gamma_{f}=\left(f, \operatorname{Id}_{Y}\right): Y \rightarrow X \times Y$ is the transpose of the graph of $f$, which in this case is a regular embedding of codimension $n$. It is straightforward to check that $\psi(a)$ is a bivariant class and $\psi$ is a group homomorphism.

For each $a \in B_{n-p} X$, one has $\varphi(\psi(a))=\psi(a)\left(1_{X}\right)=\gamma_{\operatorname{Id}_{X}}^{*}\left(a \times 1_{X}\right)=\operatorname{Id}_{X}^{*} a=$ $a \in B_{n-p} X$, so $\varphi \circ \psi$ is the identity. Given any $c \in B^{p}(X \rightarrow X)$, any morphism $f: Y \rightarrow X$ and any class $\alpha \in B_{m} Y$, one has $\psi(\varphi(c))(\alpha)=\psi\left(c\left(1_{X}\right)\right)(\alpha)=$ $\gamma_{f}^{*}\left(c\left(1_{X}\right) \times \alpha\right)=\gamma_{f}^{*}\left(c\left(1_{X} \times \alpha\right)\right)=c\left(\gamma_{f}^{*}\left(1_{X} \times \alpha\right)\right)=c(\alpha) \in B_{m-p} Y$, so $\psi \circ \varphi$ is also the identity.

To verify the compatibility with multiplication, we show that for arbitrary classes $a \in B_{n-p}(X)$ and $b \in B_{n-q}(X)$ we have $\psi(a \cdot b)=\psi(a) \cdot \psi(b) \in B^{p+q}(X \rightarrow X)$. Indeed, given any morphism $f: Y \rightarrow X$ and any class $\theta \in B_{m} Y$, we have

$$
\begin{aligned}
\psi(a \cdot b)(\theta) & =\gamma_{f}^{*}\left(\gamma_{\operatorname{Id}_{X}}^{*}(a \times b) \times \theta\right)=\left(f, f, \operatorname{Id}_{Y}\right)^{*}(a \times b \times \theta) \\
& =\gamma_{f}^{*}\left(\left(\operatorname{Id}_{X}, \gamma_{f}\right)^{*}(a \times b \times \theta)\right)=\gamma_{f}^{*}\left(a \times \gamma_{f}^{*}(b \times \theta)\right) \\
& =\psi(a)(\psi(b)(\theta))=(\psi(a) \cdot \psi(b))(\theta) \in B_{m-p-q} Y .
\end{aligned}
$$

The element $1_{X} \in B^{0}(X \rightarrow X)$ acts as multiplicative identity, hence the cap product with it maps $1_{X} \in B^{0}(X)$ to itself.

Let $f: X \rightarrow Y$ be an lci morphism between pure-dimensional smooth schemes. Naturality of $\varphi$ with respect to pullback by $f$ is equivalent to the identity

$$
f^{*}(c) \cap 1_{X}=f^{*}\left(c \cap 1_{Y}\right)
$$

for any $c \in B^{*}(Y \rightarrow Y)$, which holds by $\left(\mathrm{C}_{3}\right)$ since $1_{X}=f^{*}\left(1_{Y}\right)$.

Notation 3.3. We will set $B^{*}(X)=B^{*}\left(\operatorname{Id}_{X}: X \rightarrow X\right)$ for an arbitrary scheme $X$ in $\mathscr{C}$. Proposition 3.2 shows that this contravariant functor on $\mathscr{C}$, when restricted to the category of smooth schemes and lci morphisms, is isomorphic to the functor $B^{*}$ defined in Section 2 C.

Remark 3.4. In his construction of a bivariant theory associated to Chow theory, Fulton [1998, Chapter 17] used the Chow theory versions of our axioms $\left(\mathrm{C}_{1}\right),\left(\mathrm{C}_{2}\right)$ and $\left(\mathrm{C}_{3}\right)$, namely, compatibility of the bivariant classes with proper pushforwards, flat pullbacks and refined Gysin homomorphisms, but there is no explicit requirement of our axiom $\left(\mathrm{C}_{4}\right)$, compatibility with exterior products. This axiom $\left(\mathrm{C}_{4}\right)$ does not appear explicitly in Fulton's construction because, in the case of Chow theory $A_{*}$, axioms $\left(\mathrm{C}_{1}\right)$ and $\left(\mathrm{C}_{2}\right)$ imply axiom $\left(\mathrm{C}_{4}\right)$. More generally, this is true for any ROBM prehomology theory $B_{*}$ for which, for every $X$ in $\mathscr{C}$, the group $B_{*}(X)$ is generated by the projective pushforward images of the classes $1_{Y}$ for all smooth varieties $Y$ 
with a projective map to $X$. Indeed, using $\left(\mathrm{C}_{1}\right)$, one reduces $\left(\mathrm{C}_{4}\right)$ to the case where both $Y^{\prime}$ and $Z$ are smooth varieties, and $\alpha=1_{Y^{\prime}}$ and $\beta=1_{Z}$. In that case, from $\left(\mathrm{C}_{2}\right)$ one obtains that $c_{g h}^{(m+l)}\left(1_{Y^{\prime}} \times 1_{Z}\right)=c_{g h}^{(m+l)}\left(1_{Y^{\prime} \times Z}\right)=c_{g h}^{(m+l)}\left(h^{*} 1_{Y^{\prime}}\right)=$ $h^{\prime *} c_{g}^{(m)}\left(1_{Y^{\prime}}\right)=c_{g}^{(m)}\left(1_{Y^{\prime}}\right) \times 1_{Z}$, and then $\left(\mathrm{C}_{4}\right)$ holds in general for $B_{*}$.

\section{The equivariant version of an ROBM prehomology theory}

In this section we fix an ROBM prehomology theory $B_{*}$ on the category $\operatorname{Sch}_{k}$, and construct its equivariant version $B_{*}^{G}$, which is an ROBM prehomology theory on $G-\mathrm{Sch}_{k}$. The construction of $B_{*}^{G}$ generalizes to arbitrary ROBM prehomology theories similar constructions in Chow theory by Totaro [1999] and Edidin and Graham [1998], and in algebraic cobordism by Krishna [2012] and Heller and Malagón-López [2013].

We will need to assume throughout this section that the field $k$ is infinite and the theory $B_{*}$ satisfies the homotopy property $(\mathrm{H})$ and the localization property $(\mathrm{L})$ :

- (H) Let $p: E \rightarrow X$ be a vector bundle of rank $r$ over $X$ in $\operatorname{Sch}_{k}$. Then $p^{*}$ : $B_{*}(X) \rightarrow B_{*+r}(E)$ is an isomorphism.

- (L) For any closed immersion $i: Z \rightarrow X$ with open complement $j: U=$ $X \backslash Z \rightarrow X$, the following sequence is exact:

$$
B_{*}(Z) \stackrel{i_{*}}{\longrightarrow} B_{*}(X) \stackrel{j^{*}}{\longrightarrow} B_{*}(U) \longrightarrow 0 .
$$

4A. Algebraic groups, quotients and good systems of representations. Let $G$ be a linear algebraic group. If $X$ is a scheme with a $G$-action $\sigma: G \times X \rightarrow X$ and the geometric quotient of $X$ by the action of $G$ exists, it will be denoted by $X \rightarrow X / G$. When the geometric quotient $\pi: X \rightarrow X / G$ exists, it is called a principal $G$-bundle if the morphism $\pi$ is faithfully flat and the morphism $\psi=\left(\sigma, \operatorname{pr}_{X}\right): G \times X \rightarrow$ $X \times_{X / G} X$ is an isomorphism. By [Mumford et al. 1994, Proposition 0.9], if $G$ acts freely on $U \in G$-Sch $k$ and the geometric quotient $\pi: U \rightarrow U / G$ exists in $\operatorname{Sch}_{k}$, for some quasiprojective scheme $U / G$, then $\pi: U \rightarrow U / G$ is a principal $G$-bundle. Moreover, by [Mumford et al. 1994, Proposition 7.1], for any $X \in G-\mathrm{Sch}_{k}$ the geometric quotient $\pi^{\prime}: X \times U \rightarrow(X \times U) / G$ also exists in $\operatorname{Sch}_{k}$, it is a principal $G$-bundle and $(X \times U) / G$ is quasiprojective. In this case we denote the scheme $(X \times U) / G$ by $X \times{ }^{G} U$.

Definition 4.1. We say that $\left\{\left(V_{i}, U_{i}\right)\right\}_{i \in \mathbb{Z}^{+}}$is a good system of representations of $G$ if each $V_{i}$ is a $G$-representation, $U_{i} \subseteq V_{i}$ is a $G$-invariant open subset and they satisfy the following conditions:

(1) $G$ acts freely on $U_{i}$ and $U_{i} / G$ exists as a quasiprojective scheme in $\operatorname{Sch}_{k}$.

(2) For each $i$ there is a $G$-representation $W_{i}$ so that $V_{i+1}=V_{i} \oplus W_{i}$. 
(3) $U_{i} \oplus\{0\} \subseteq U_{i+1}$ and the inclusion factors as $U_{i}=U_{i} \oplus\{0\} \subseteq U_{i} \oplus W_{i} \subseteq U_{i+1}$.

(4) $\operatorname{codim}_{V_{i}}\left(V_{i} \backslash U_{i}\right)<\operatorname{codim}_{V_{j}}\left(V_{j} \backslash U_{j}\right)$ for $i<j$.

For any algebraic group $G$ there exist good systems of representations (see [Totaro 1999, Remark 1.4]). The following lemma lists some basic facts regarding the properties of morphisms induced on geometric quotients:

Lemma 4.2. Let $f: X \rightarrow Y$ be a $G$-equivariant morphism in $G$-Sch ${ }_{k}$, and let $\left\{\left(V_{i}, U_{i}\right)\right\}$ be a good system of representations of $G$.

(1) For each $i$ the quotient $X \times{ }^{G} U_{i}=\left(X \times U_{i}\right) / G$ exists in $\operatorname{Sch}_{k}$ and it is quasiprojective. The induced morphisms $\phi_{i j}: X \times{ }^{G} U_{i} \rightarrow X \times{ }^{G} U_{j}$ are lci morphisms for $j \geq i$. If $X$ is smooth then $X \times{ }^{G} U_{i}$ is also smooth.

(2) Let $\mathbf{P}$ be one of the following properties of morphisms: open immersion, closed immersion, regular embedding, projective, smooth, lci. If $f: X \rightarrow Y$ satisfies the property $\mathbf{P}$ in the category $G-\operatorname{Sch}_{k}$, then the induced maps $f_{i}: X \times{ }^{G} U_{i} \rightarrow$ $Y \times{ }^{G} U_{i}$ satisfy property $\mathbf{P}$ in the category $\operatorname{Sch}_{k}$.

(3) For any morphisms $g: Y \rightarrow X$ and $f: Z \rightarrow X$ in $G-\operatorname{Sch}_{k}$ and any indices $j \geq i$, the square diagrams
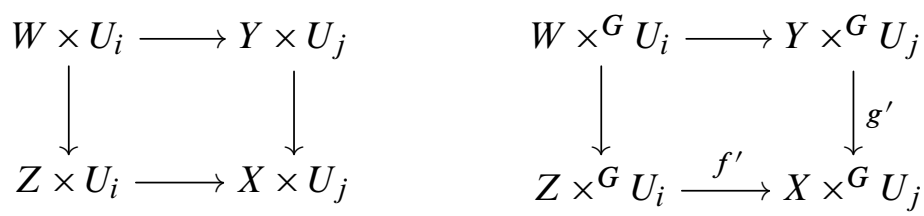

induced by the Cartesian product $W=Y \times_{X} Z$ are fiber squares, and furthermore they are Tor-independent if $f$ and $g$ are Tor-independent.

Proof. For proofs of the assertions in (1) and (2) see [Edidin and Graham 1998, Proposition 2] and [Heller and Malagón-López 2013, 2.2.2, Lemma 9]. For (3), given any $T \in \operatorname{Sch}_{k}$ and morphisms $g^{\prime \prime}: T \rightarrow Y \times{ }^{G} U_{j}$ and $f^{\prime \prime}: T \rightarrow Z \times{ }^{G} U_{i}$ such that $f^{\prime} \circ f^{\prime \prime}=g^{\prime} \circ g^{\prime \prime}$, we let $G$ act on $\tilde{T}=T \times{ }_{\left(X \times{ }^{G} U_{j}\right)}\left(X \times U_{j}\right)$ via the morphism $G \times \widetilde{T} \rightarrow \widetilde{T}$ induced by the product of the trivial action of $G$ on $T$ and the action of $G$ on $X \times U_{j}$. By [Mumford et al. 1994, Amplification 7.1], $G$-equivariant morphisms from $\widetilde{T}$ to each of $X \times U_{j}, Y \times U_{j}, Z \times U_{i}$ and $W \times U_{i}$ correspond to the morphisms induced on the quotients from $T$ to $X \times{ }^{G} U_{j}, Y \times{ }^{G} U_{j}, Z \times{ }^{G} U_{i}$ and $W \times{ }^{G} U_{i}$, respectively. The assertion that the squares in (3) are Cartesian follows easily from these observations. If $f$ and $g$ are Tor-independent, then $\operatorname{Tor}_{m}^{O_{X}}\left(\mathrm{O}_{Y}, \mathrm{O}_{Z}\right)=0$ for all $m>0$, and clearly $\operatorname{Tor}_{m}^{\mathrm{O}_{j}}\left(\mathrm{O}_{U_{j}}, \mathrm{O}_{U_{i}}\right)=0$ for all $m>0$. Hence, by applying locally the spectral sequence associated to the double complex obtained as the tensor product of two complexes, we obtain that $\operatorname{Tor}_{m}^{\mathrm{O}_{X} \times U_{j}}\left(\mathrm{O}_{Y \times U_{j}}, \mathrm{O}_{Z \times U_{i}}\right)=0$ for all $m>0$. Since $Y \times U_{j}=\left(Y \times{ }^{G} U_{j}\right) \times{ }_{\left(X \times{ }^{G} U_{j}\right)}\left(X \times U_{j}\right)$ and $Z \times U_{i}=$ 
$\left(Z \times{ }^{G} U_{i}\right) \times\left(X \times{ }^{G} U_{j}\right)\left(X \times U_{j}\right)$, by faithfully flat base change for Tor $_{m}$ we have that $\operatorname{Tor}_{m}^{{ }^{X} \times{ }^{G} U_{j}}\left({ }^{O} Y_{\times}{ }^{G} U_{j},{ }{ }_{Z \times G} U_{i}\right)=0$ for all $m>0$. Therefore, in this case the given squares are also Tor-independent.

4B. Construction of $\boldsymbol{B}_{*}^{\boldsymbol{G}}(\boldsymbol{X})$. Fix a good system of representations $\left\{\left(V_{i}, U_{i}\right)\right\}$ of $G$. For any scheme $X \in G-\operatorname{Sch}_{k}$, define $B_{*}^{G}(X)=\bigoplus_{n \in \mathbb{Z}} B_{n}^{G}(X)$, where

$$
B_{n}^{G}(X)=\lim _{i} B_{n+\operatorname{dim} U_{i}-\operatorname{dim} G}\left(X \times{ }^{G} U_{i}\right)
$$

To simplify notation, we will often write

$$
B_{*}^{G}(X)=\lim _{i} B_{*}\left(X \times{ }^{G} U_{i}\right),
$$

where the limit is taken in each degree separately. Equivalently, the limit is taken in the category of graded abelian groups, with $B_{*}\left(X \times{ }^{G} U_{i}\right)$ having grading shifted so that the maps in the inverse system are homogeneous of degree zero.

To see that $B_{*}^{G}$ is independent of the choice of a good system of representations, one can formally follow the argument presented in the case of algebraic cobordism in [Heller and Malagón-López 2013, Proposition 15 and Theorem 16], which we outline below for the reader's convenience. The proof of the next proposition requires the field $k$ to be infinite (this is used in the proof to construct a local section of the projection $\left.\pi\right|_{U}: U \rightarrow X$ ).

Proposition 4.3. Assume that the ROBM prehomology theory $B_{*}$ satisfies properties $(\mathrm{H})$ and $(\mathrm{L})$. Let $\pi: E \rightarrow X$ be a vector bundle over a scheme $X$ of rank $r$. Let $U \subseteq E$ be an open subscheme with closed complement $S=E \backslash U$.

(1) If $X$ is affine and $\operatorname{codim}_{E} S>\operatorname{dim} X$ then $\left.\pi\right|_{U} ^{*}: B_{m}(X) \rightarrow B_{m+r}(U)$ is an isomorphism for all $m$.

(2) For $X$ arbitrary, there is an integer $n(X)$ depending only on $X$, such that $\left.\pi\right|_{U} ^{*}: B_{m}(X) \rightarrow B_{m+r}(U)$ is an isomorphism for all $m$ whenever $\operatorname{codim}_{E} S>$ $n(X)$.

Proof. The case when $B_{*}$ is algebraic cobordism is [Heller and Malagón-López 2013, Proposition 15]. The proof given there only uses the formal properties of algebraic cobordism as an ROBM prehomology theory satisfying $(\mathrm{H})$ and $(\mathrm{L})$, so it translates formally to the present setting.

Proposition 4.4. For any $X \in G-\mathrm{Sch}_{k}, B_{*}^{G}(X)$ is independent of the choice of a good system of representations of $G$ up to canonical isomorphism.

Proof. We use Bogomolov's double filtration argument. Assume that $\left\{\left(V_{i}, U_{i}\right)\right\}$ and $\left\{\left(V_{i}^{\prime}, U_{i}^{\prime}\right)\right\}$ are good systems of representations of $G$. For a fixed index $i$, since $G$ acts freely on $U_{i}$, it also acts freely on $U_{i} \times V_{j}^{\prime}$ and $U_{i} \times U_{j}^{\prime}$ for all $j$. Hence 
$X \times{ }^{G}\left(U_{i} \times V_{j}^{\prime}\right) \rightarrow X \times{ }^{G} U_{i}$ is a vector bundle. By Proposition 4.3(2) there is an integer $N_{i}$ such that the lci pullbacks induce canonical isomorphisms

$$
B_{*}\left(X \times{ }^{G} U_{i}\right) \cong B_{*}\left(X \times{ }^{G}\left(U_{i} \times U_{j}^{\prime}\right)\right)
$$

for each $j \geq N_{i}$. Therefore, there is a canonical isomorphism

$$
\varliminf_{i} B_{*}\left(X \times{ }^{G} U_{i}\right)=\lim _{i} \lim _{j} B_{*}\left(X \times{ }^{G}\left(U_{i} \times U_{j}^{\prime}\right)\right)
$$

Similarly, exchanging the role of the good systems of representations we obtain a canonical isomorphism

$$
\varliminf_{j} B_{*}\left(X \times{ }^{G} U_{j}^{\prime}\right)=\varliminf_{j} \lim _{i} B_{*}\left(X \times \times^{G}\left(U_{i} \times U_{j}^{\prime}\right)\right) .
$$

To obtain the conclusion, we only need to observe that the right-hand sides of (4-1) and (4-2) are canonically isomorphic to the inverse limit of the system $\left\{B_{*}\left(X \times{ }^{G}\left(U_{i} \times U_{j}^{\prime}\right)\right)\right\}_{i, j}$, where the maps

$$
B_{*}\left(X \times{ }^{G}\left(U_{i} \times U_{j}^{\prime}\right)\right) \rightarrow B_{*}\left(X \times{ }^{G}\left(U_{i^{\prime}} \times U_{j^{\prime}}^{\prime}\right)\right)
$$

are the corresponding lci pullbacks for all $i \geq i^{\prime}$ and $j \geq j^{\prime}$.

4C. The induced ROBM prehomology theory structure on $\boldsymbol{B}_{*}^{\boldsymbol{G}}$. We now show that the ROBM prehomology structure of the theory $B_{*}$ induces an ROBM prehomology structure on $B_{*}^{G}$. We use functoriality of the inverse limit to construct projective pushforwards, smooth pullbacks, and exterior and intersection products on $B_{*}^{G}$. To define a homomorphism between two inverse limits, we construct a map between the two inverse systems.

Fix a good system of representations $\left\{\left(V_{i}, U_{i}\right)\right\}$ of $G$. Given any projective $G$-equivariant morphism $f: X \rightarrow Y$, the morphisms $\left\{f_{i}: X \times{ }^{G} U_{i} \rightarrow Y \times{ }^{G} U_{i}\right\}$ are projective. The fiber square on the left in (4-3) is Tor-independent for any $j \geq i$,

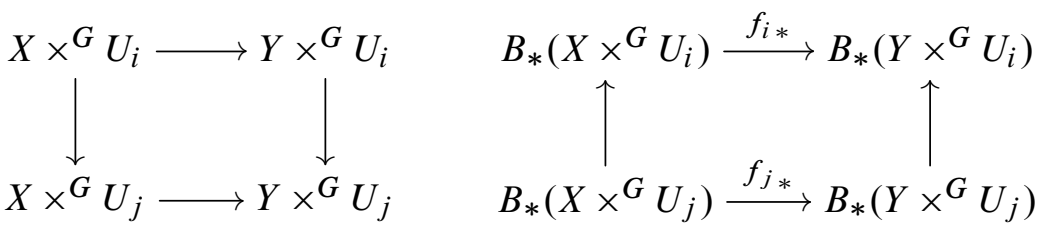

hence the square on the right in (4-3) is commutative for any $j \geq i$. Hence, the maps $f_{i *}$ induce a homomorphism between the limits $f_{*}: B_{*}^{G}(X) \rightarrow B_{*}^{G}(Y)$.

Smooth pullbacks are defined in a similar way. For intersection products, given a $G$-equivariant lci morphism $f: Z \rightarrow X$ of codimension $d$ and any $G$-equivariant morphism $g: Y \rightarrow X$, with $W=Z \times_{X} Y$, first we apply the operation $\times{ }^{G} U_{i}$ to the whole intersection product diagram. The result is again an intersection product 
diagram. The two fiber squares on the first line of (4-4) are Tor-independent for any $j \geq i$ :

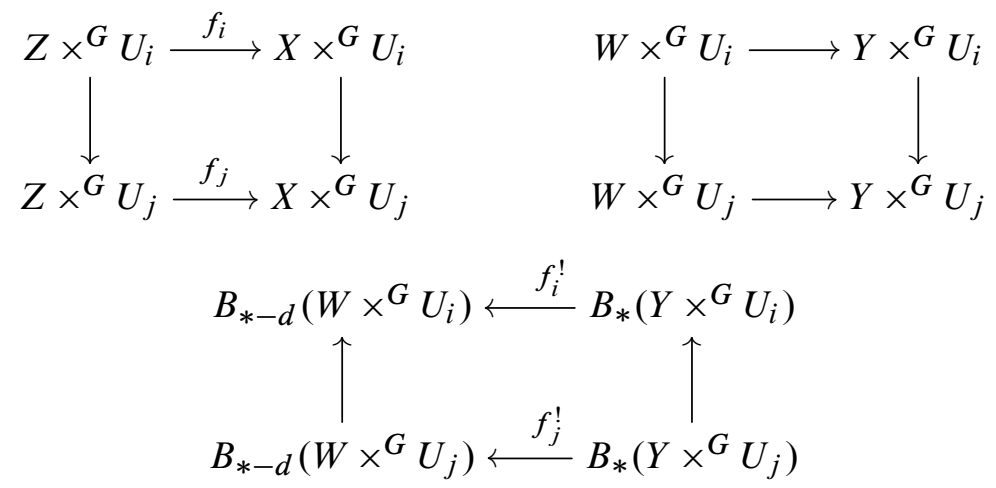

hence the square on the bottom line of (4-4) is commutative for any $j \geq i$. Hence, the maps $f_{i}^{!}$induce a homomorphism between the limits $f^{!}: B_{*}^{G}(Y) \rightarrow B_{*-d}^{G}(W)$.

To define the exterior product, note that the morphisms

$$
\phi_{i}:(X \times Y) \times^{G}\left(U_{i} \times U_{i}\right) \rightarrow\left(X \times^{G} U_{i}\right) \times\left(Y \times{ }^{G} U_{i}\right)
$$

are smooth (see [Borel 1991, Theorem 6.8]). We compose the associated smooth pullback and the exterior product of $B_{*}$ to get

$$
\begin{aligned}
\times_{i}: B_{*}\left(X \times{ }^{G} U_{i}\right) \times B_{*}\left(Y \times{ }^{G} U_{i}\right) \rightarrow B_{*}\left(\left(X \times{ }^{G} U_{i}\right) \times\left(Y \times{ }^{G} U_{i}\right)\right) \\
\rightarrow B_{*}\left((X \times Y) \times{ }^{G}\left(U_{i} \times U_{i}\right)\right) .
\end{aligned}
$$

The morphisms $x_{i}$ are compatible with maps in the inverse systems, and hence they define the exterior product map between limits:

$$
\times: B_{*}^{G} X \times B_{*}^{G} Y \rightarrow B_{*}^{G}(X \times Y) .
$$

The elements $1_{U_{i} / G} \in B_{*}\left(\operatorname{Spec} k \times{ }^{G} U_{i}\right)$ define $1 \in B_{*}^{G}(X)$.

It remains to prove that the theory $B_{*}^{G}$ with the operations defined above satisfies the axioms of an ROBM prehomology theory. Each axiom amounts to a statement about the commutativity of a diagram of homomorphisms. One can check that in each case the commutativity holds at each level $i$, and hence it also holds in the limit. Using the double filtration argument as before, one can also check that these projective pushforwards, smooth pullbacks, exterior and intersection products are independent of the good system of representations.

The conclusions of this section can then be summarized as: 
Theorem 4.5. The functor $B_{*}^{G}$ with the projective pushforwards, smooth pullbacks, and exterior and intersection products constructed above is a refined oriented BorelMoore prehomology theory on the category $G-\mathrm{Sch}_{k}$. We call $B_{*}^{G}$ the equivariant version of $B_{*}$.

4D. Operational equivariant theory. For a given ROBM prehomology theory $B_{*}$ on $\mathrm{Sch}_{k}$, we constructed an associated equivariant version $B_{*}^{G}$ as an ROBM prehomology theory on $G-\operatorname{Sch}_{k}$. The construction of Section 3 applied to $B_{*}$ and to $B_{*}^{G}$ produces operational bivariant theories $B^{*}$ on $\operatorname{Sch}_{k}$ and $\left(B^{G}\right)^{*}$ on $G-\operatorname{Sch}_{k}$ respectively. We denote $\left(B^{G}\right)^{*}$ by $B_{G}^{*}$ and call it the operational equivariant version of $B_{*}$

One can switch the order of the two steps in the construction of $B_{G}^{*}$ and define an "equivariant operational" theory

$$
\widetilde{B}_{G}^{*}(X)=\lim _{i} B^{*}\left(X \times{ }^{G} U_{i}\right) .
$$

The two theories $B_{G}^{*}(X)$ and $\widetilde{B}_{G}^{*}(X)$ turn out to be isomorphic if we assume that $B_{*}$ satisfies the descent property (D) described in the next section. This property was first proved by Gillet [1984] in the case of Chow groups and $K$-theory. It has several other consequences for ROBM prehomology theories that are studied in the next section.

\section{Descent sequences}

We assume in this section that the field $k$ has characteristic zero, or, more generally, we assume that every scheme $X$ in $\operatorname{Sch}_{k}$ or in $G-\mathrm{Sch}_{k}$ has a smooth projective (equivariant) envelope $\pi: \tilde{X} \rightarrow X$ as defined in Section 5A. We fix an ROBM prehomology theory $B_{*}$ on one of the categories $\operatorname{Sch}_{k}$ or $G-\operatorname{Sch}_{k}$ and consider the following property (D):

- (D) For any envelope $\pi: \tilde{X} \rightarrow X$, with $\pi$ projective, the sequence

$$
B_{*}\left(\tilde{X} \times_{X} \tilde{X}\right) \stackrel{p_{1 *}-p_{2 *}}{\longrightarrow} B_{*}(\tilde{X}) \stackrel{\pi_{*}}{\longrightarrow} B_{*}(X) \longrightarrow 0,
$$

is exact, where $p_{i}: \tilde{X} \times_{X} \tilde{X} \rightarrow \tilde{X}$ is the projection on the $i$-th factor for $i=1,2$.

5A. Envelopes. An envelope of a scheme $X$ in $\operatorname{Sch}_{k}$ is a proper morphism $\pi$ : $\tilde{X} \rightarrow X$ such that for every subvariety $V$ of $X$ there is a subvariety $\tilde{V}$ of $\tilde{X}$ that is mapped birationally onto $V$ by $\pi$. If $G$ is an algebraic group, a $G$-equivariant envelope of a scheme $X$ in $G-\operatorname{Sch}_{k}$ is a proper $G$-equivariant morphism $\pi: \tilde{X} \rightarrow X$ such that for every $G$-invariant subvariety $V$ of $X$ there is a $G$-invariant subvariety $\tilde{V}$ of $\tilde{X}$ that is mapped birationally onto $V$ by $\pi$. 
In the following, an envelope in the category $\operatorname{Sch}_{k}$ means an ordinary envelope and an envelope in the category $G-\mathrm{Sch}_{k}$ means a $G$-equivariant envelope. If $\pi: \tilde{X} \rightarrow X$ is an envelope, we say that it is a smooth envelope if $\tilde{X}$ is smooth, and we say that it is a projective envelope if $\pi$ is a projective morphism. Likewise, we say that the envelope $\pi: \tilde{X} \rightarrow X$ is birational if, for some dense open subset $U$ of $X, \pi$ induces an isomorphism $\pi \mid: \pi^{-1}(U) \rightarrow U$. The composition of envelopes is again an envelope and the fiber product of an envelope by any morphism is again an envelope.

The domain $\tilde{X}$ of an envelope is not required to be connected; hence, if we assume that varieties over $k$ admit (equivariant) resolutions of singularities via a projective morphism, then by induction on the dimension it follows easily that for every scheme $X$ in $\operatorname{Sch}_{k}$ (respectively in $G-\mathrm{Sch}_{k}$ ) there exists a smooth projective birational (equivariant) envelope $\pi: \widetilde{X} \rightarrow X$.

Notice that if $\pi: \widetilde{X} \rightarrow X$ is an envelope in $G-\operatorname{Sch}_{k}$ and if $\tilde{U} \in G-\operatorname{Sch}_{k}$ has a free $G$-action such that $\widetilde{U} / G$ exists as a quasiprojective scheme in $\operatorname{Sch}_{k}$, then the induced morphism $\pi_{G}: \tilde{X} \times^{G} \tilde{U} \rightarrow X \times^{G} \tilde{U}$ is an envelope in $\operatorname{Sch}_{k}$. Furthermore if $\pi$ is either a smooth, projective or birational envelope, then $\pi_{G}$ is a smooth, projective or birational envelope, respectively. Moreover, if $\pi \mid: \pi^{-1}(U) \rightarrow U$ is an isomorphism for some $G$-invariant open subset $U$ of $X$ and $\left\{Z_{i}\right\}$ are $G$-invariant closed subschemes of $X$ such that $X \backslash U=\bigcup Z_{i}$, then $\pi_{G}$ maps the open subset $\pi_{G}^{-1}\left(U \times{ }^{G} \tilde{U}\right)=\pi^{-1}(U) \times{ }^{G} \tilde{U}$ of $\tilde{X} \times{ }^{G} \tilde{U}$ isomorphically onto the open subset $U \times^{G} \tilde{U}$ of $X \times{ }^{G} \tilde{U}$, and the closed subschemes $\left\{Z_{i} \times{ }^{G} \tilde{U}\right\}$ of $X \times^{G} \tilde{U}$ satisfy that $(X \backslash U) \times^{G} \tilde{U}=\left(X \times^{G} \tilde{U}\right) \backslash\left(U \times{ }^{G} \tilde{U}\right)=\bigcup\left(Z_{i} \times{ }^{G} \tilde{U}\right)$.

5B. Operational equivariant versus equivariant operational. Assume now that the theory $B_{*}$ on $\mathrm{Sch}_{k}$ satisfies properties $(\mathrm{H})$ and $(\mathrm{L})$, and we can thus define the operational equivariant theory $B_{G}^{*}$ as well as the "equivariant operational" theory $\widetilde{B}_{G}^{*}$ as in Section 4D. We show that if $B_{*}$ also satisfies property (D), then these two bivariant theories are isomorphic. For simplicity, we prove this isomorphism only for the bivariant cohomology theory $B_{G}^{*}(X)$.

Lemma 5.1. Let $B_{*}$ be an ROBM prehomology theory on $\mathrm{Sch}_{k}$ that satisfies $(\mathrm{H})$, (L) and (D). Then, for any scheme $X \in G-\mathrm{Sch}_{k}$ and any projective envelope $\pi: \tilde{X} \rightarrow X$, the pushforward homomorphism $\pi_{*}: B_{*}^{G}(\tilde{X}) \rightarrow B_{*}^{G}(X)$ is surjective.

Proof. Recall that the inverse limit $\lim$ is a left exact functor from the category of inverse systems of (graded) abelian groups. Given a short exact sequence of inverse systems

$$
0 \longrightarrow\left(E_{i}\right) \longrightarrow\left(F_{i}\right) \longrightarrow\left(G_{i}\right) \longrightarrow 0,
$$

the sequence of limits

$$
0 \longrightarrow \lim _{\longleftarrow} E_{i} \longrightarrow \lim _{\longleftarrow} F_{i} \longrightarrow \lim _{\longleftarrow} G_{i} \longrightarrow 0
$$


is exact if the system $\left(E_{i}\right)$ satisfies the Mittag-Leffler condition. This condition is satisfied, for example, if the maps $E_{i} \rightarrow E_{j}$ for $i>j$ in the inverse system are all surjective.

Given an exact sequence

$$
\left(E_{i}\right) \longrightarrow\left(F_{i}\right) \longrightarrow\left(G_{i}\right) \longrightarrow 0,
$$

one may replace the system $\left(E_{i}\right)$ with its image $\left(I_{i}\right)$ in $\left(F_{i}\right)$ to get a short exact sequence. If all maps in the inverse system $\left(E_{i}\right)$ are surjective, then they are also surjective in $\left(I_{i}\right)$, and it follows that the map of limits $\lim _{\longleftarrow} F_{i} \rightarrow \underset{\lim }{\longleftarrow} G_{i}$ is surjective.

We apply the previous discussion to the sequence of inverse systems

$$
B_{*}\left(\left(\tilde{X} \times_{X} \tilde{X}\right) \times{ }^{G} U_{i}\right) \longrightarrow B_{*}\left(\tilde{X} \times{ }^{G} U_{i}\right) \longrightarrow B_{*}\left(X \times{ }^{G} U_{i}\right) \longrightarrow 0 .
$$

This sequence is exact by property (D): the map $\tilde{X} \times^{G} U_{i} \rightarrow X \times^{G} U_{i}$ is an envelope and

$$
\left(\tilde{X} \times_{X} \tilde{X}\right) \times{ }^{G} U_{i} \cong\left(\tilde{X} \times{ }^{G} U_{i}\right) \times_{X \times{ }^{G} U_{i}}\left(\tilde{X} \times{ }^{G} U_{i}\right) .
$$

For any scheme $Y$ in $G$-Sch $k$, the lci pullbacks

$$
B_{*}\left(Y \times{ }^{G} U_{i}\right) \rightarrow B_{*}\left(Y \times{ }^{G} U_{j}\right)
$$

are surjective for all $i>j$. This follows from properties (H) and (L) because the inclusion $Y \times{ }^{G} U_{j} \rightarrow Y \times{ }^{G} U_{i}$ can be factored as the inclusion of the zero section of a vector bundle followed by an open immersion. Applying this to the case $Y=\tilde{X} \times_{X} \tilde{X}$ gives the statement of the lemma.

Proposition 5.2. If the ROBM prehomology theory $B_{*}$ on $\mathrm{Sch}_{k}$ satisfies properties $(\mathrm{H}),(\mathrm{L})$ and $(\mathrm{D})$, then for any $X$ in $G-\mathrm{Sch}_{k}$ there exists an isomorphism

$$
B_{G}^{*}(X) \cong \lim _{i} B^{*}\left(X \times{ }^{G} U_{i}\right) .
$$

The isomorphism is natural with respect to pullback by any morphism $f: X^{\prime} \rightarrow X$ in $G-\mathrm{Sch}_{k}$.

Proof. Given $X$ in $G-S \operatorname{ch}_{k}$ we define a homomorphism

$$
\varphi_{X}: \lim _{i} B^{*}\left(X \times{ }^{G} U_{i}\right) \rightarrow B_{G}^{*}(X)
$$

as follows: given $c=\left(c_{i}\right) \in \lim _{i} B^{*}\left(X \times{ }^{G} U_{i}\right)$, where $c_{i} \in B^{*}\left(X \times{ }^{G} U_{i}\right)$ for each $i$, and given any $G$-equivariant morphism $f: Y \rightarrow X$ and a class $\alpha=$ $\left(\alpha_{i}\right) \in B_{*}^{G}(Y)=\lim _{i} B_{*}\left(Y \times{ }^{G} U_{i}\right)$, where $\alpha_{i} \in B_{*}\left(Y \times{ }^{G} U_{i}\right)$ for each $i$, one sets $\varphi_{X}(c)(\alpha)=\left(c_{i}\left(\alpha_{i}\right)\right) \in \lim _{i} B_{*}\left(Y \times{ }^{G} U_{i}\right)=B_{*}^{G}(Y)$. It is straightforward to verify that $\varphi_{X}(c)$ is well-defined and is indeed a bivariant class in $B_{*}^{G}(X)$, so that $\varphi_{X}$ is a well-defined homomorphism.

It is clear from the definitions that $\varphi_{X}$ is natural with respect to pullbacks. 
We prove that $\varphi_{X}$ is an isomorphism. First, we consider the case when $X$ is smooth. In this case, each $X \times{ }^{G} U_{i}$ is smooth as well. By Poincare duality (Proposition 3.2) we have isomorphisms

$$
B_{G}^{*}(X) \stackrel{\cap 1_{X}}{\longrightarrow} B_{*}^{G}(X) \text { and } B^{*}\left(X \times{ }^{G} U_{i}\right) \stackrel{\cap 1_{X \times} U_{i}}{\longrightarrow} B_{*}\left(X \times{ }^{G} U_{i}\right)
$$

for each $i$. Passing to the component-wise inverse limit and composing appropriately, one obtains the isomorphism

$\lim _{i} B^{*}\left(X \times{ }^{G} U_{i}\right) \stackrel{\left(\cap 1_{X \times G}{ }_{U_{i}}\right)_{i}}{\longrightarrow} \lim _{i} B_{*}\left(X \times{ }_{\varphi_{X}}^{G} U_{i}\right)=B_{*}^{G}(X) \stackrel{\left(\cap 1_{X}\right)^{-1}}{\longrightarrow} B_{G}^{*}(X)$

which can be verified directly to be $\varphi_{X}$.

For the general case, given $X$ we choose a $G$-equivariant envelope $\pi: \tilde{X} \rightarrow X$ so that $\pi$ is projective and $\widetilde{X}$ is smooth. Let $\pi_{i}: \tilde{X} \times{ }^{G} U_{i} \rightarrow X \times{ }^{G} U_{i}$ be the induced morphisms. We get a commutative diagram

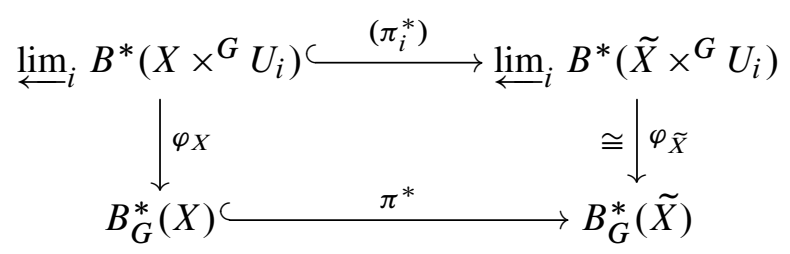

We claim that $\pi^{*}$ and $\left(\pi_{i}^{*}\right)$ are injective. To see this, assume that $\pi^{*} c=0$ for some $c \in B_{G}^{*}(X)$. Given a $G$-equivariant map $f: Y \rightarrow X$ and a class $\alpha \in B_{*}^{G}(Y)$, form the fiber product

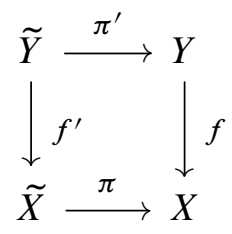

with morphisms as indicated. Since $\pi^{\prime}$ is an envelope, by Lemma 5.1 there exists $\tilde{\alpha} \in B_{*}^{G}(\tilde{Y})$ such that $\pi_{*}^{\prime}(\tilde{\alpha})=\alpha$. We have $c(\alpha)=c\left(\pi_{*}^{\prime}(\tilde{\alpha})\right)=\pi_{*}^{\prime}(c(\tilde{\alpha}))=$ $\pi_{*}^{\prime}\left(\left(\pi^{*} c\right)(\tilde{\alpha})\right)=0$, and it follows that $\pi^{*}$ is injective. Since each $\pi_{i}$ is also an envelope, the same argument proves that the $\pi_{i}^{*}$ are injective, and then so is the component-wise inverse limit homomorphism $\left(\pi_{i}^{*}\right)$. In particular, it follows that $\varphi_{X}$ is injective, as $\varphi_{\tilde{X}}$ is an isomorphism by the smooth case.

To prove the surjectivity of $\varphi_{X}$, we consider $c \in B_{G}^{*}(X)$, and construct an element mapping to $\varphi_{\tilde{X}}^{-1}\left(\pi^{*} c\right)$ by $\left(\pi_{i}^{*}\right)$. Let

$$
p_{j}: \tilde{X} \times_{X} \tilde{X} \rightarrow \tilde{X} \quad \text { and } \quad p_{j}^{\prime}:\left(\tilde{X} \times{ }^{G} U_{i}\right) \times_{X \times{ }^{G} U_{i}}\left(\tilde{X} \times{ }^{G} U_{i}\right) \rightarrow \tilde{X} \times{ }^{G} U_{i}
$$


be the projections on the corresponding $j$-th factor for $j=1,2$. Let $\left(\tilde{c}_{i}\right) \in$ $\lim _{i} B^{*}\left(\tilde{X} \times{ }^{G} U_{i}\right)$ be the image of $c$ under $\varphi_{\tilde{X}}^{-1} \circ \pi^{*}$, where $\tilde{c}_{i} \in B^{*}\left(\tilde{X} \times{ }^{G} U_{i}\right)$ for all $i$. Now, using that $\varphi_{\tilde{X} \times X} \tilde{X}$ is injective and that $\left(p_{1}^{*}-p_{2}^{*}\right)\left(\pi^{*} c\right)=0$, it follows that $\left(p_{1}^{\prime *}-p_{2}^{\prime *}\right)\left(\tilde{c}_{i}\right)=0$ for each $i$. We define a class $\left(c_{i}\right) \in \varliminf_{i} B^{*}\left(X \times{ }^{G} U_{i}\right)$ as follows: given a morphism $f: Y \rightarrow X \times{ }^{G} U_{i}$ and a class $\alpha \in \overleftarrow{B_{*}}(Y)$, form the fiber product

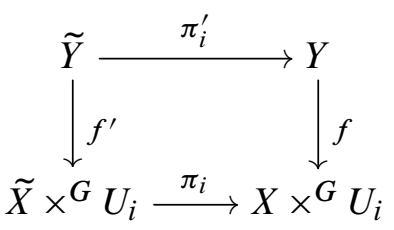

with morphisms as indicated, and set $c_{i}(\alpha)=\pi_{i *}^{\prime}\left(\tilde{c}_{i}(\tilde{\alpha})\right)$, where $\tilde{\alpha} \in B_{*}(\tilde{Y})$ is any class satisfying $\pi_{i *}^{\prime}(\tilde{\alpha})=\alpha$ (which exists since $\pi_{i}^{\prime}$ is an envelope). To see that $c_{i}(\alpha)$ is independent of the choice of $\tilde{\alpha}$, it is enough to see that $\pi_{i *}^{\prime}\left(\tilde{c}_{i}(\beta)\right)=0$ for each class $\beta \in B_{*}(\tilde{Y})$ such that $\pi_{i *}^{\prime} \beta=0$. By property (D), given such a class $\beta$ there exists a class $\gamma \in B_{*}\left(\tilde{Y} \times_{Y} \tilde{Y}\right)$ such that $\beta=g_{1 *}(\gamma)-g_{2 *}(\gamma)$, where $g_{j}: \tilde{Y} \times_{Y} \tilde{Y} \rightarrow \tilde{Y}$ are the projections for $j=1$, 2. Since $\pi_{i}^{\prime} \circ g_{1}=\pi_{i}^{\prime} \circ g_{2}$, it follows that $\pi_{i *}^{\prime}\left(\tilde{c}_{i}(\beta)\right)=\pi_{i *}^{\prime}\left(\tilde{c}_{i}\left(g_{1 *}(\gamma)-g_{2 *}(\gamma)\right)\right)=\left(\left(\pi_{i}^{\prime} \circ g_{1}\right)_{*}-\left(\pi_{i}^{\prime} \circ g_{2}\right)_{*}\right)\left(\tilde{c}_{i}(\gamma)\right)=0$, and then $c_{i}$ is well-defined. It is straightforward to verify that each $c_{i}$ satisfies conditions $\left(\mathrm{C}_{1}\right)-\left(\mathrm{C}_{4}\right)$, so they define bivariant classes $c_{i} \in B^{*}\left(X \times{ }^{G} U_{i}\right)$. Moreover, it is clear that the classes $c_{i}$ agree under the bivariant pullbacks $B^{*}\left(X \times{ }^{G} U_{j}\right) \rightarrow$ $B^{*}\left(X \times{ }^{G} U_{i}\right)$ for each $j \geq i$, so they define a class $\left(c_{i}\right) \in{\underset{\lim }{i}}_{i} B^{*}\left(X \times{ }^{G} U_{i}\right)$. To prove that $\varphi_{X}$ is surjective, it is enough to see that $\pi_{i}^{*} c_{i}=\tilde{c}_{i}$ for each $i$. For this, let $f: Y \rightarrow \tilde{X} \times{ }^{G} U_{i}$ be any morphism and form the fiber diagram

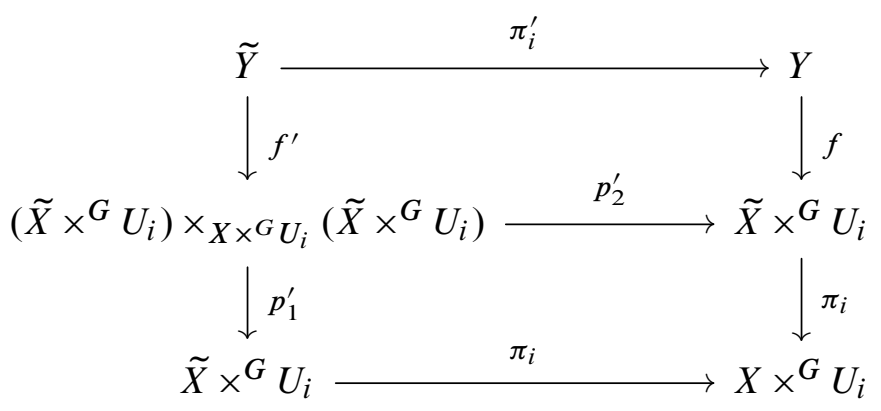

with morphisms as indicated. Consider a class $\alpha \in B_{*}(Y)$ and any class $\tilde{\alpha} \in B_{*}(\tilde{Y})$ such that $\pi_{i *}^{\prime}(\tilde{\alpha})=\alpha$. Since $p_{1}^{\prime *}\left(\tilde{c}_{i}\right)=p_{2}^{\prime *}\left(\tilde{c}_{i}\right)$, we have that

$$
\begin{aligned}
\left(\pi_{i}^{*} c_{i}\right)_{f}(\alpha) & =\pi_{i *}^{\prime}\left(\left(\tilde{c}_{i}\right)_{p_{1}^{\prime} \circ f^{\prime}}(\tilde{\alpha})\right)=\pi_{i *}^{\prime}\left(\left(f^{\prime *} p_{1}^{\prime *} \tilde{c}_{i}\right)(\tilde{\alpha})\right) \\
& =\pi_{i *}^{\prime}\left(\left(f^{\prime *} p_{2}^{\prime *} \tilde{c}_{i}\right)(\tilde{\alpha})\right)=\pi_{i *}^{\prime}\left(\left(\tilde{c}_{i}\right)_{f \circ \pi_{i}^{\prime}}(\tilde{\alpha})\right)=\left(\tilde{c}_{i}\right)_{f}(\alpha) .
\end{aligned}
$$

Hence $\pi_{i}^{*} c_{i}=\tilde{c}_{i}$ for each $i$, and the proof is complete. 
5C. Kimura-type descent sequence for bivariant theories and inductive computation of bivariant groups. Theorem 5.3 and Theorem 5.6 below were proved by Kimura [1992] in the case of Chow theory $A_{*}$. We generalize his proofs to arbitrary ROBM prehomology theories.

Theorem 5.3. Let $B_{*}$ be an ROBM prehomology theory on $\mathscr{b}=\operatorname{Sch}_{k}$ or $\mathscr{b}=$ $G-\mathrm{Sch}_{k}$ that satisfies property (D). Let $\pi: \tilde{X} \rightarrow X$ be a projective envelope in $\mathscr{C}$, $Y \rightarrow X$ a morphism in $\mathscr{C}$, and $\tilde{Y}=\tilde{X} \times_{X} Y$. Then the following sequence is exact:

$$
0 \rightarrow B^{*}(Y \rightarrow X) \stackrel{\pi^{*}}{\longrightarrow} B^{*}(\tilde{Y} \rightarrow \tilde{X}) \stackrel{p_{1}^{*}-p_{2}^{*}}{\longrightarrow} B^{*}\left(\tilde{Y} \times_{Y} \tilde{Y} \rightarrow \tilde{X} \times_{X} \tilde{X}\right) .
$$

Proof. Assume that $\pi^{*} c=0$ for some $c \in B^{*}(Y \rightarrow X)$. Given a morphism $f: X^{\prime} \rightarrow X$ and a class $\alpha \in B_{*}\left(X^{\prime}\right)$, form the fiber diagram on the left in (5-3):
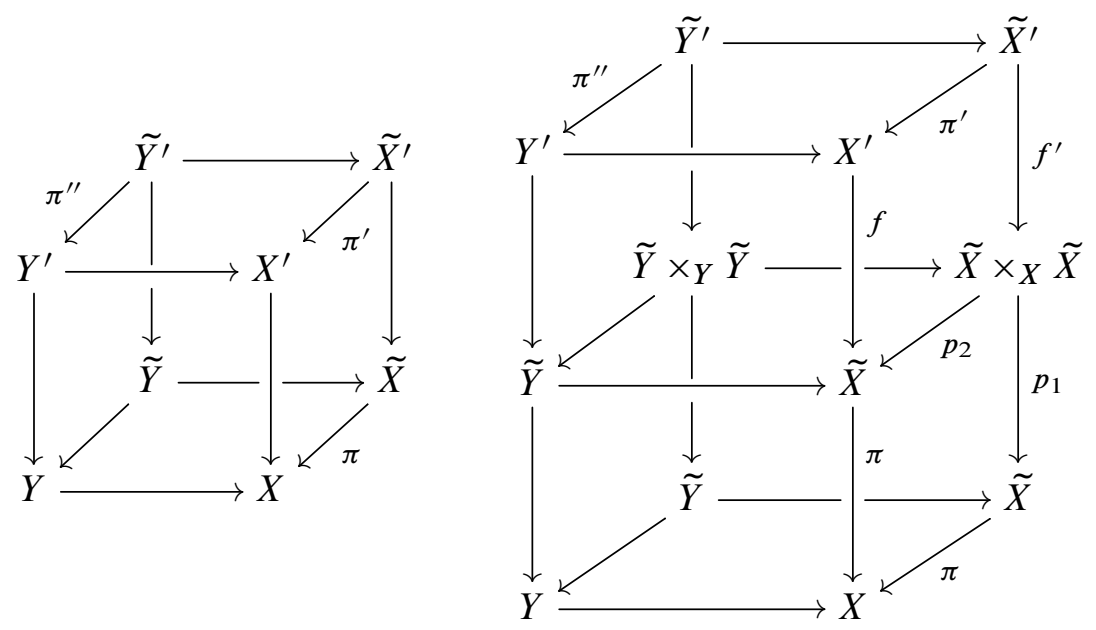

with morphisms labeled as indicated. Since $\pi^{\prime}$ is an envelope, there exists $\tilde{\alpha} \in$ $B_{*}\left(\tilde{X}^{\prime}\right)$ such that $\pi_{*}^{\prime}(\tilde{\alpha})=\alpha$. We have that $c(\alpha)=c\left(\pi_{*}^{\prime}(\tilde{\alpha})\right)=\pi_{*}^{\prime \prime}(c(\tilde{\alpha}))=$ $\pi_{*}^{\prime \prime}\left(\left(\pi^{*} c\right)(\tilde{\alpha})\right)=0$, and it follows that $\pi^{*}$ is injective. By the functoriality of pullbacks it follows that $\left(p_{1}^{*}-p_{2}^{*}\right) \circ \pi^{*}=0$.

Now, let $\tilde{c} \in B^{*}(\tilde{Y} \rightarrow \tilde{X})$ be a bivariant class such that $\left(p_{1}^{*}-p_{2}^{*}\right)(\tilde{c})=0$. We define a class $c \in B^{*}(Y \rightarrow X)$ as follows: given a morphism $f: X^{\prime} \rightarrow X$ and a class $\alpha \in B_{*}\left(X^{\prime}\right)$, form once again the fiber diagram on the left in (5-3), with morphisms as indicated, and set $c(\alpha)=\pi_{*}^{\prime \prime}(\tilde{c}(\tilde{\alpha}))$, where $\tilde{\alpha} \in B_{*}\left(\tilde{X}^{\prime}\right)$ is any class satisfying $\pi_{*}^{\prime}(\tilde{\alpha})=\alpha$ (which exists since $\pi^{\prime}$ is an envelope). To see that $c(\alpha)$ is independent of the choice of $\tilde{\alpha}$, it is enough to see that $\pi_{*}^{\prime \prime}(\tilde{c}(\beta))=0$ for each class $\beta \in B_{*}\left(\tilde{X}^{\prime}\right)$ such that $\pi_{*}^{\prime} \beta=0$. Let $g_{j}: \tilde{X}^{\prime} \times_{X^{\prime}} \tilde{X}^{\prime} \rightarrow \tilde{X}^{\prime}$ and $g_{j}^{\prime}: \tilde{Y}^{\prime} \times_{Y^{\prime}} \tilde{Y}^{\prime} \rightarrow \tilde{Y}^{\prime}$ be the projections for $j=1,2$. By property (D), given such a class $\beta$ there exists a class $\gamma \in B_{*}\left(\tilde{X}^{\prime} \times X_{X^{\prime}} \tilde{X}^{\prime}\right)$ such that $\beta=g_{1 *}(\gamma)-g_{2 *}(\gamma)$. Since $\pi^{\prime \prime} \circ g_{1}^{\prime}=\pi^{\prime \prime} \circ g_{2}^{\prime}$, it follows that $\pi_{*}^{\prime \prime}(\tilde{c}(\beta))=\pi_{*}^{\prime \prime}\left(\tilde{c}\left(g_{1 *}(\gamma)-g_{2 *}(\gamma)\right)\right)=\left(\left(\pi^{\prime \prime} \circ g_{1}^{\prime}\right)_{*}-\left(\pi^{\prime \prime} \circ g_{2}^{\prime}\right)_{*}\right)(\tilde{c}(\gamma))=0$, 
and then $c$ is well-defined. It is straightforward to verify that $c$ satisfies the conditions $\left(\mathrm{C}_{1}\right)-\left(\mathrm{C}_{4}\right)$, so this construction yields a bivariant class $c \in B_{*}(Y \rightarrow X)$. To finish the proof we show that $\pi^{*} c=\tilde{c}$. For this, let $f: X^{\prime} \rightarrow \tilde{X}$ be any morphism and consider a class $\alpha \in B_{*}\left(X^{\prime}\right)$ and any class $\tilde{\alpha} \in B_{*}\left(\tilde{X}^{\prime}\right)$ such that $\pi_{*}^{\prime}(\tilde{\alpha})=\alpha$. Form the fiber diagram on the right in (5-3) with morphisms as indicated. Since $p_{1}^{*}(\tilde{c})=p_{2}^{*}(\tilde{c})$, we have that

$$
\begin{aligned}
\left(\pi^{*} c\right)_{f}(\alpha) & =\pi_{*}^{\prime \prime}\left(\tilde{c}_{p_{1} \circ f^{\prime}}(\tilde{\alpha})\right)=\pi_{*}^{\prime \prime}\left(\left(f^{\prime *} p_{1}^{*} \tilde{c}\right)(\tilde{\alpha})\right) \\
& =\pi_{*}^{\prime \prime}\left(\left(f^{*} p_{2}^{*} \tilde{c}\right)(\tilde{\alpha})\right)=\pi_{*}^{\prime \prime}\left(\tilde{c}_{f \circ \pi^{\prime}}(\tilde{\alpha})\right)=\tilde{c}_{f}(\alpha) .
\end{aligned}
$$

Hence $\pi^{*} c=\tilde{c}$, and the proof is complete.

Corollary 5.4. Let $B_{*}$ be an ROBM prehomology theory on $\mathscr{C}=\operatorname{Sch}_{k}$ or $\mathscr{C}=$ $G-\mathrm{Sch}_{k}$ that satisfies property (D). Then for any projective envelope $\pi: \widetilde{X} \rightarrow X$ in $\mathscr{C}$ the following sequence is exact:

$$
0 \rightarrow B^{*}(X) \stackrel{\pi^{*}}{\longrightarrow} B^{*}(\tilde{X}) \stackrel{p_{1}^{*}-p_{2}^{*}}{\longrightarrow} B^{*}\left(\tilde{X} \times_{X} \tilde{X}\right) .
$$

The following lemma is a simple consequence of the localization property (L) or of the property (D).

Lemma 5.5. Let $B_{*}$ be an ROBM prehomology theory on $\mathscr{C}=\operatorname{Sch}_{k}$ or $\mathscr{C}=G-\mathrm{Sch}_{k}$ that satisfies either property (L) or property (D). If $X=\bigcup_{i=1}^{r} Z_{i}$, where each $f_{i}: Z_{i} \rightarrow X$ is a closed subscheme of $X$, then

$$
B_{*}(X)=\sum_{i=1}^{r} f_{i *}\left(B_{*}\left(Z_{i}\right)\right)
$$

Proof. The general case follows at once from the case $r=2$, so we assume that $X=Z_{1} \cup Z_{2}$. If $B_{*}$ satisfies (L), we have that the sequence

$$
B_{*}\left(Z_{1}\right) \stackrel{f_{1 *}}{\longrightarrow} B_{*}(X) \stackrel{\left.f_{2}\right|^{*}}{\longrightarrow} B_{*}\left(X \backslash Z_{1}\right) \longrightarrow 0 .
$$

is exact. By the compatibility of pullbacks and pushforwards and using localization, it is clear that $\left.f_{2}\right|^{*}$ maps $f_{2 *}\left(B_{*}\left(Z_{2}\right)\right)$ onto $B_{*}\left(X \backslash Z_{1}\right)$. It is clear now that $B_{*}(X)=f_{1 *}\left(B_{*}\left(Z_{1}\right)\right)+f_{2 *}\left(B_{*}\left(Z_{2}\right)\right)$, as desired. If $B_{*}$ satisfies (D), the result follows since $B_{*}$ is additive and the projective morphism from the disjoint union $Z_{1} \amalg Z_{2} \rightarrow X$ induced by the inclusions is an envelope.

The following result can be proved as a corollary of Theorem 5.3. It gives an inductive method for computing bivariant groups: 
Theorem 5.6. Let $B_{*}$ be an ROBM prehomology theory on $\mathscr{b}=\operatorname{Sch}_{k}$ or $\mathscr{C}=$ $G-\operatorname{Sch}_{k}$. Let $\pi: \tilde{X} \rightarrow X$ be a projective and birational envelope in $\mathscr{C}$. Let $Y \rightarrow X$ be a morphism in $\mathscr{C}$ and $\tilde{Y}=\tilde{X} \times_{X} Y$. Assume that $B_{*}$ satisfies the conclusion of Lemma 5.5 and that the sequence (5-2) in Theorem 5.3 is exact (e.g., it is enough to assume that $B_{*}$ satisfies (D)). Let $\pi: \pi^{-1}(U) \stackrel{\sim}{\longrightarrow} U$ for some open dense $U \subset X$. Let $S_{i} \subset X$ be closed subschemes such that $X \backslash U=\bigcup S_{i}$. Let $E_{i}=\pi^{-1}\left(S_{i}\right)$, and let $\pi_{i}: E_{i} \rightarrow S_{i}$ be the induced morphism. Then for a class $\tilde{c} \in B^{*}(\tilde{Y} \rightarrow \widetilde{X})$ the following are equivalent:

(1) $\tilde{c}=\pi^{*}(c)$ for some $c \in B^{*}(Y \rightarrow X)$.

(2) For all $i,\left.\tilde{c}\right|_{E_{i}}=\pi_{i}^{*}\left(c_{i}\right)$ for some $c_{i} \in B^{*}\left(Y \times_{X} S_{i} \rightarrow S_{i}\right)$.

Proof. If $\tilde{c}=\pi^{*}(c)$ for some $c \in B^{*}(Y \rightarrow X)$, then by the functoriality of pullbacks $\left.\tilde{c}\right|_{E_{i}}=\pi_{i}^{*}\left(\left.c\right|_{S_{i}}\right)$ for all $i$, and then (2) holds if we take $c_{i}=\left.c\right|_{S_{i}} \in B^{*}\left(Y \times_{X} S_{i} \rightarrow S_{i}\right)$. Reciprocally, assume that there are classes $c_{i}$ as in (2). Let $p_{1}, p_{2}: \widetilde{X} \times_{X} \widetilde{X} \rightarrow \widetilde{X}$ be the projections. By Theorem 5.3 it is enough to show that $p_{1}^{*} \tilde{c}=p_{2}^{*} \tilde{c}$, i.e., that for any morphism $f: Z \rightarrow \tilde{X} \times_{X} \tilde{X}$ and for any class $\alpha \in B_{*} Z$ we have that $\left(p_{1}^{*} \tilde{c}\right)(\alpha)=\left(p_{2}^{*} \tilde{c}\right)(\alpha)$. Let $f_{i}^{\prime}: E_{i} \times_{X} E_{i} \rightarrow \tilde{X} \times_{X} \tilde{X}$ be the corresponding closed embeddings and let $\Delta: \tilde{X} \rightarrow \tilde{X} \times_{X} \tilde{X}$ be the diagonal morphism which is also a closed embedding. Notice that $\tilde{X} \times_{X} \tilde{X}$ is the union the closed subschemes $\Delta(\tilde{X})$ and $f_{i}^{\prime}\left(E_{i} \times_{X} E_{i}\right)$ for all $i$. Let $Z_{0}=f^{-1}(\Delta(\tilde{X}))$ and $Z_{i}=f^{-1}\left(f_{i}^{\prime}\left(E_{i} \times_{X} E_{i}\right)\right)$, with inclusions $\Delta^{\prime}: Z_{0} \rightarrow Z$ and $f_{i}^{\prime \prime}: Z_{i} \rightarrow Z$ for each $i$. Then, by Lemma 5.5, in order to prove that $\left(p_{1}^{*} \tilde{c}\right)(\alpha)=\left(p_{2}^{*} \tilde{c}\right)(\alpha)$, we can assume that either $\alpha=f_{i *}^{\prime \prime}\left(\alpha_{i}\right)$ for some $i$ and some $\alpha_{i} \in B_{*}\left(Z_{i}\right)$ or $\alpha=\Delta_{*}^{\prime}\left(\alpha_{0}\right)$ for some $\alpha_{0} \in B_{*}\left(Z_{0}\right)$. In the first case, for $j=1$ and $j=2$ consider the fiber diagram

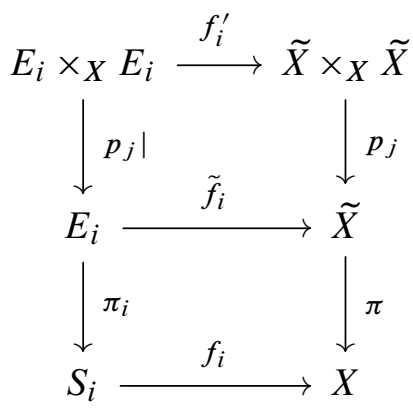

with morphisms as labeled. Let $\Delta^{\prime \prime}: Z_{0} \times_{X} Y \rightarrow Z \times_{X} Y$ and $g_{i}^{\prime \prime}: Z_{i} \times_{X} Y \rightarrow Z \times_{X} Y$ be the morphisms obtained from $\Delta^{\prime}$ and $f_{i}^{\prime \prime}$ by base change. We have

$$
\begin{aligned}
\left(p_{j}^{*} \tilde{c}\right)(\alpha) & =\left(p_{j}^{*} \tilde{c}\right)\left(f_{i *}^{\prime \prime}\left(\alpha_{i}\right)\right)=g_{i *}^{\prime \prime}\left(\left(p_{j}^{*} \tilde{c}\right)\left(\alpha_{i}\right)\right)=g_{i *}^{\prime \prime}\left(\tilde{c}\left(\alpha_{i}\right)\right) \\
& =g_{i *}^{\prime \prime}\left(\left(\tilde{f}_{i}^{*} \tilde{c}\right)\left(\alpha_{i}\right)\right)=g_{i *}^{\prime \prime}\left(\left(\pi_{i}^{*} c_{i}\right)\left(\alpha_{i}\right)\right)=g_{i *}^{\prime \prime}\left(\left(\left.p_{j}\right|^{*} \pi_{i}^{*} c_{i}\right)\left(\alpha_{i}\right)\right) \\
& =g_{i *}^{\prime \prime}\left(\left(\left(\pi_{i} \circ p_{j} \mid\right)^{*} c_{i}\right)\left(\alpha_{i}\right)\right) .
\end{aligned}
$$


Since $\pi_{i} \circ p_{1}\left|=\pi_{i} \circ p_{2}\right|$, it follows that in the first case $\left(p_{1}^{*} \tilde{c}\right)(\alpha)=\left(p_{2}^{*} \tilde{c}\right)(\alpha)$. In the second case, for $j=1$ and $j=2$ we have

$$
\begin{aligned}
\left(p_{j}^{*} \tilde{c}\right)(\alpha) & =\left(p_{j}^{*} \tilde{c}\right)\left(\Delta_{*}^{\prime}\left(\alpha_{0}\right)\right)=\Delta_{*}^{\prime \prime}\left(\left(p_{j}^{*} \tilde{c}\right)\left(\alpha_{0}\right)\right)=\Delta_{*}^{\prime \prime}\left(\left(\Delta^{*} p_{j}^{*} \tilde{c}\right)\left(\alpha_{0}\right)\right) \\
& =\Delta_{*}^{\prime \prime}\left(\left(\left(p_{j} \circ \Delta\right)^{*} \tilde{c}\right)\left(\alpha_{0}\right)\right)=\Delta_{*}^{\prime \prime}\left(\left(\operatorname{Id}_{\tilde{X}}^{*} \tilde{c}\right)\left(\alpha_{0}\right)\right)=\Delta_{*}^{\prime \prime}\left(\tilde{c}\left(\alpha_{0}\right)\right)
\end{aligned}
$$

Therefore, in the second case $\left(p_{1}^{*} \tilde{c}\right)(\alpha)=\left(p_{2}^{*} \tilde{c}\right)(\alpha)$, and the proof is complete.

5D. Kimura-type descent sequence for bivariant equivariant theories and inductive computation of bivariant equivariant groups. Theorem 5.3 and Theorem 5.6 proved above can be applied to the equivariant theory $B_{*}^{G}$, provided that it satisfies property (D). We cannot prove property (D) for $B_{*}^{G}$ assuming that it holds for $B_{*}$. We will therefore give a different proof of the statements of Theorem 5.3 and Theorem 5.6 for $B_{*}^{G}$ that depends only on $B_{*}$ satisfying property (D). We give proofs for the bivariant cohomology groups $B_{G}^{*}(X)$ only.

Theorem 5.7. Let $B_{*}$ be an ROBM prehomology theory on $\mathrm{Sch}_{k}$ that satisfies properties (H), (L) and (D). Let $\pi: \widetilde{X} \rightarrow X$ be a projective envelope in $G-\mathrm{Sch}_{k}$, and let the terminology be as in Theorem 5.6.

(a) The following sequence is exact:

$$
0 \rightarrow B_{G}^{*}(X) \stackrel{\pi^{*}}{\longrightarrow} B_{G}^{*}(\tilde{X}) \stackrel{p_{1}^{*}-p_{2}^{*}}{\longrightarrow} B_{G}^{*}\left(\tilde{X} \times_{X} \tilde{X}\right) .
$$

(b) If $\pi$ is also birational, then for a class $\tilde{c} \in B_{G}^{*}(\tilde{X})$ the following are equivalent:

(1) $\tilde{c}=\pi^{*}(c)$ for some $c \in B_{G}^{*}(X)$.

(2) For all $i,\left.\tilde{c}\right|_{E_{i}}=\pi_{i}^{*}\left(c_{i}\right)$ for some $c_{i} \in B_{G}^{*}\left(S_{i}\right)$.

Proof. (a) Since for each $i$ the map $\tilde{X} \times{ }^{G} U_{i} \rightarrow X \times{ }^{G} U_{i}$ is an envelope, by Corollary 5.4 the sequence

$$
0 \longrightarrow B^{*}\left(X \times{ }^{G} U_{i}\right) \longrightarrow B^{*}\left(\tilde{X} \times{ }^{G} U_{i}\right) \longrightarrow B^{*}\left((\tilde{X} \times X \tilde{X}) \times^{G} U_{i}\right)
$$

is exact. Applying the left exact functor lim and using Proposition 5.2 gives the desired result.

(b) In view of part (a), the conclusion follows from Theorem 5.6 if we show that the ROBM prehomology theory $B_{*}^{G}$ satisfies the conclusion of Lemma 5.5.

For this, it is enough to consider the case $r=2$, so we let $X=Z_{1} \cup Z_{2}$ and let $Z=Z_{1} \sqcup Z_{2}$. The projective morphism $Z \rightarrow X$ induced by the inclusions is an envelope; hence $B_{*}^{G}(Z) \rightarrow B_{*}^{G}(X)$ is surjective by Lemma 5.1 


\section{An overview of algebraic cobordism theory}

In this section we recall the definition and main properties of algebraic cobordism $\Omega_{*}$. This theory was constructed by Levine and Morel [2007]. Later, Levine and Pandharipande [2009] found a geometric presentation of the cobordism groups. We will use the construction of [Levine and Pandharipande 2009] as the definition, but refer to [Levine and Morel 2007] for its properties. This construction and the proofs of some of the facts stated below use resolution of singularities, factorization of birational maps and some Bertini-type theorems; thus, we will assume for the remainder of this article that the field $k$ has characteristic zero.

The equivariant algebraic cobordism $\Omega_{*}^{G}$ was constructed first by Krishna [2012] and by Heller and Malagón-López [2013]. Krishna and Uma [2013] showed how to compute the equivariant and ordinary cobordism groups of smooth toric varieties; we will recall their result in Section 7.

For $X$ in $\operatorname{Sch}_{k}$, let $M(X)$ be the set of isomorphism classes of projective morphisms $f: Y \rightarrow X$ for $Y \in \operatorname{Sm}_{k}$. This set is a monoid under disjoint union of the domains; let $\mathcal{M}^{+}(X)$ be its group completion. The elements of $\mathcal{M}^{+}(X)$ are called cycles. The class of $f: Y \rightarrow X$ in $\mathcal{M}^{+}(X)$ is denoted $[f: Y \rightarrow X]$. The group $\mathcal{M}^{+}(X)$ is free abelian, generated by the cycles $[f: Y \rightarrow X]$ where $Y$ is irreducible.

A double point degeneration is a morphism $\pi: Y \rightarrow \mathbb{P}^{1}$, with $Y \in \mathrm{Sm}_{k}$ of pure dimension, such that $Y_{\infty}=\pi^{-1}(\infty)$ is a smooth divisor on $Y$ and $Y_{0}=\pi^{-1}(0)$ is a union $A \cup B$ of smooth divisors intersecting transversely along $D=A \cap B$. Define

$$
\mathbb{P}_{D}=\mathbb{P}\left(\mathscr{O}_{D}(A) \oplus \mathscr{O}_{D}\right)=\operatorname{Proj}_{\mathscr{O}_{D}}\left(\operatorname{Sym}_{\mathscr{O}_{D}}^{*}\left(\mathscr{O}_{D}(A) \oplus \mathscr{O}_{D}\right)\right),
$$

where $\mathscr{O}_{D}(A)$ denotes $\left.\mathscr{O}_{Y}(A)\right|_{D}$. (Notice that $\mathbb{P}\left(\mathscr{O}_{D}(A) \oplus \mathscr{O}_{D}\right) \cong \mathbb{P}\left(\mathscr{O}_{D}(B) \oplus \mathscr{O}_{D}\right)$ because $O_{D}(A+B) \cong O_{D}$.

Let $X \in \mathrm{Sch}_{k}$, and let $Y \in \mathrm{Sm}_{k}$ have pure dimension. Let $p_{1}, p_{2}$ be the two projections of $X \times \mathbb{P}^{1}$. A double point relation is defined by a projective morphism $\pi: Y \rightarrow X \times \mathbb{P}^{1}$ such that $p_{2} \circ \pi: Y \rightarrow \mathbb{P}^{1}$ is a double point degeneration. Let

$$
\left[Y_{\infty} \rightarrow X\right], \quad[A \rightarrow X], \quad[B \rightarrow X], \quad\left[\mathbb{P}_{D} \rightarrow X\right]
$$

be the cycles obtained by composing with $p_{1}$. The double point relation is

$$
\left[Y_{\infty} \rightarrow X\right]-[A \rightarrow X]-[B \rightarrow X]+\left[\mathbb{P}_{D} \rightarrow X\right] \in \mathcal{M}^{+}(X) .
$$

Let $\mathscr{R}(X)$ be the subgroup of $\mathcal{M}^{+}(X)$ generated by all the double point relations. The cobordism group of $X$ is defined to be

$$
\Omega_{*}(X)=\mu^{+}(X) / \mathscr{R}(X) .
$$


The group $\mathcal{M}^{+}(X)$ is graded so that $[f: Y \rightarrow X]$ lies in degree $\operatorname{dim} Y$ when $Y$ has pure dimension. Since double point relations are homogeneous, this grading gives a grading on $\Omega_{*}(X)$. We write $\Omega_{n}(X)$ for the degree- $n$ part of $\Omega_{*}(X)$.

There is a functorial pushforward homomorphism $f_{*}: \Omega_{*}(X) \rightarrow \Omega_{*}(Z)$ for $f: X \rightarrow Z$ projective, and a functorial pullback homomorphism $g^{*}: \Omega_{*}(Z) \rightarrow$ $\Omega_{*+d}(X)$ for $g: X \rightarrow Z$ a smooth morphism of relative dimension $d$. These homomorphisms are both defined on the cycle level; the pullback does not preserve grading. The exterior product on $\Omega_{*}(X)$ is also defined on the cycle level:

$$
[Y \rightarrow X] \times[Z \rightarrow W]=[Y \times Z \rightarrow X \times W] .
$$

Levine and Morel [2007] presented a construction of functorial pullbacks $g^{*}$ along lci morphisms $g$, and, more generally, of refined lci pullbacks.

The groups $\Omega_{*}(X)$ with these projective pushforward, lci pullback and exterior products form an oriented Borel-Moore homology theory (see [Levine and Morel 2007]). Moreover, with those refined lci pullbacks it is also an ROBM prehomology theory (see [Levine and Morel 2007]).

As in the case of a general ROBM prehomology theory, $\Omega_{*}$ (Spec $k$ ) is a ring, $\Omega_{*}(X)$ is a module over $\Omega_{*}(\operatorname{Spec} k)$ for general $X$, and $\Omega_{*}(X)$ is an algebra over $\Omega_{*}(\operatorname{Spec} k)$ for smooth $X$. When $X$ is smooth and has pure dimension, we also use the cohomological notation

$$
\Omega^{*}(X)=\Omega_{\operatorname{dim} X-*}(X) .
$$

Then $\Omega^{*}(X)$ is a graded algebra over the graded ring $\Omega^{*}(\operatorname{Spec} k)$. The class $1_{X}=\left[\operatorname{Id}_{X}: X \rightarrow X\right]$ is the identity of the algebra. Similar conventions are used for the equivariant cobordism groups.

Remark 6.1. Algebraic cobordism satisfies the homotopy property $(H)$ and the localization property (L) [Levine and Morel 2007], as well as the descent property (D) [González and Karu 2015]. It follows that everything proved in the previous sections for general ROBM prehomology theories can be applied to the algebraic cobordism theory. This includes the construction of the equivariant cobordism theory $\Omega_{*}^{G}$, the operational cobordism theory $\Omega^{*}$ and the operational equivariant cobordism theory $\Omega_{G}^{*}$. This also includes the descent exact sequences for the operational theories $\Omega^{*}$ and $\Omega_{G}^{*}$ and the inductive method for their computation using envelopes.

The following part of Theorem 5.7 applied to $\Omega_{G}^{*}(X)$ will be used in the next section:

Theorem 6.2. Assume that $\pi: \tilde{X} \rightarrow X$ is a projective birational envelope in $G-\operatorname{Sch}_{k}$, with $\pi: \pi^{-1}(U) \stackrel{\sim}{\rightarrow} U$ for some open nonempty $G$-equivariant $U \subset X$. Let $S_{i} \subset X$ be closed $G$-equivariant subschemes, such that $X \backslash U=\bigcup S_{i}$. Let $E_{i}=\pi^{-1}\left(S_{i}\right)$, 
and let $\pi_{i}: E_{i} \rightarrow S_{i}$ be the induced morphism. Then $\pi^{*}: \Omega_{G}^{*}(X) \rightarrow \Omega_{G}^{*}(\tilde{X})$ is injective, and for a class $\tilde{c} \in \Omega_{G}^{*}(\tilde{X})$ the following are equivalent:

(1) $\tilde{c}=\pi^{*}(c)$ for some $c \in \Omega_{G}^{*}(X)$.

(2) For all $i,\left.\tilde{c}\right|_{E_{i}}=\pi_{i}^{*}\left(c_{i}\right)$ for some $c_{i} \in \Omega_{G}^{*}\left(S_{i}\right)$.

6A. Formal group law. Algebraic cobordism is endowed with first Chern class operators associated to line bundles, whose definition agrees with the one in Definition 2.9. We recall the formal group law satisfied by these operators.

A formal group law on a commutative ring $R$ is a power series $F_{R}(u, v) \in R \llbracket u, v \rrbracket$ satisfying:

(a) $F_{R}(u, 0)=F_{R}(0, u)=u$.

(b) $F_{R}(u, v)=F_{R}(v, u)$.

(c) $F_{R}\left(F_{R}(u, v), w\right)=F_{R}\left(u, F_{R}(v, w)\right)$.

Thus

$$
F_{R}(u, v)=u+v+\sum_{i, j>0} a_{i, j} u^{i} v^{j},
$$

where the $a_{i, j} \in R$ satisfy $a_{i, j}=a_{j, i}$ and some additional relations coming from property (c). We think of $F_{R}$ as giving a formal addition

$$
u+F_{R} v=F_{R}(u, v) .
$$

There exists a unique power series $\chi(u) \in R \llbracket u \rrbracket$ such that $F_{R}(u, \chi(u))=0$. Set $[-1]_{F_{R}} u=\chi(u)$. Composing $F_{R}$ and $\chi$, we can form linear combinations

$$
\left[n_{1}\right]_{F_{R}} u_{1}+F_{R}\left[n_{2}\right]_{F_{R}} u_{2}+F_{R} \cdots+F_{R}\left[n_{r}\right]_{F_{R}} u_{r} \in R \llbracket u_{1}, \ldots, u_{r} \rrbracket
$$

for $n_{i} \in \mathbb{Z}$ and $u_{i}$ variables.

There exists a universal formal group law $F_{\mathbb{}}$, and its coefficient ring $\mathbb{L}$ is called the Lazard ring. This ring can be constructed as the quotient of the polynomial ring $\mathbb{Z}\left[A_{i, j}\right]_{i, j>0}$ by the relations imposed by the three axioms above. The images of the variables $A_{i, j}$ in the quotient ring are the coefficients $a_{i, j}$ of the formal group law $F_{[}$. It is shown in [Levine and Morel 2007] that $\Omega^{*}(\operatorname{Spec} k)$ is isomorphic as a graded ring to $\mathbb{L}$ with grading induced by letting $A_{i, j}$ have degree $-i-j+1$. The power series $F_{\mathbb{L}}(u, v)$ is then homogeneous of degree 1 if $u$ and $v$ both have degree 1 .

The formal group law on $\mathbb{L}$ describes the first Chern class operators of tensor products of line bundles:

$$
\tilde{\mathrm{c}}_{1}(L \otimes M)=F_{\mathbb{Q}}\left(\tilde{\mathrm{c}}_{1}(L), \tilde{\mathrm{c}}_{1}(M)\right)
$$

for any line bundles $L$ and $M$ on any scheme $X$ in $\operatorname{Sch}_{k}$. 


\section{Operational equivariant cobordism of toric varieties}

Let $X_{\Delta}$ be a smooth quasiprojective toric variety corresponding to a fan $\Delta$. Krishna and Uma [2013] showed that the $T$-equivariant cobordism ring of $X_{\Delta}$ is isomorphic to the ring of piecewise graded power series on the fan $\Delta$. Our goal here is to show that, for any fan $\Delta$, the ring of piecewise graded power series on $\Delta$ is isomorphic to the operational $T$-equivariant cobordism ring of $X_{\Delta}$. When $X_{\Delta}$ is smooth, this follows from the result of Krishna and Uma by Poincaré duality. For singular $X_{\Delta}$ we follow the argument of [Payne 2006] in the case of Chow theory to reduce to the smooth case.

We use the standard notation for toric varieties [Fulton 1993]. Let $N \cong \mathbb{Z}^{n}$ be a lattice. It determines a split torus $T$ with character lattice $M=\operatorname{Hom}(N, \mathbb{Z})$. A toric variety $X_{\Delta}$ is defined by a fan $\Delta$ in $N$.

Every quasiprojective toric variety $X_{\Delta}$ with torus $T$ is in the category $T-\operatorname{Sch}_{k}$, since each line bundle on such variety admits a $T$-linearization. We will write $\Omega_{*}^{T}\left(X_{\Delta}\right)$ for the $T$-equivariant cobordism group of $X_{\Delta}$. For a smooth $X_{\Delta}$ we also use the cohomological notation $\Omega_{T}^{*}\left(X_{\Delta}\right)=\Omega_{\operatorname{dim} X_{\Delta-*}}^{T}\left(X_{\Delta}\right)$. The operational $T$ equivariant cobordism ring is denoted $\Omega_{T}^{*}\left(X_{\Delta}\right)$. For smooth $X_{\Delta}$, the two definitions of $\Omega_{T}^{*}\left(X_{\Delta}\right)$ are identified via Poincaré duality.

7A. Graded power series rings. We start by recalling some notions from [Krishna and Uma 2013].

Let $A=\bigoplus_{i \in \mathbb{Z}} A_{i}$ be a commutative graded ring. The graded power series ring is

$$
A \llbracket t_{1}, t_{2}, \ldots, t_{n} \rrbracket_{\mathrm{gr}}=\bigoplus_{d \in \mathbb{Z}} S_{d} .
$$

Here $S_{d}$ is the group of degree- $d$ homogeneous power series $\sum_{I} a_{I} t^{I}$, where the sum runs over multi-indices $I=\left(i_{1}, \ldots, i_{n}\right) \in \mathbb{Z}_{\geq 0}^{n}, t^{I}=t_{1}^{i_{1}} \cdots t_{n}^{i_{n}}$, and $a_{I} \in A_{d-i_{1}-\cdots-i_{n}}$. This ring can be viewed as the inverse limit in the category of commutative graded rings

$$
A \llbracket t_{1}, t_{2}, \ldots, t_{n} \rrbracket_{\mathrm{gr}} \cong \lim _{j} A\left[t_{1}, \ldots, t_{n}\right] /\left(t_{1}^{j}, \ldots, t_{n}^{j}\right) .
$$

Let us also recall the topological tensor product. Given two inverse limits of graded $A$-modules $B=\lim _{j} B_{j}$ and $C=\lim _{j} C_{j}$, define

$$
B \widehat{\otimes}_{A} C=\varliminf_{j}\left(B_{j} \otimes_{A} C_{j}\right) .
$$

(All limits are in the category of graded $A$-modules, hence the tensor product is again a graded $A$-module.) Consider now the case where $C=A \llbracket t_{1}, t_{2}, \ldots, t_{n} \rrbracket_{\mathrm{gr}}$ and $B$ is a limit of graded $A$-algebras. We may then identify

$$
B \widehat{\otimes}_{A} A \llbracket t_{1}, t_{2}, \ldots, t_{n} \rrbracket_{\mathrm{gr}}=B \llbracket t_{1}, t_{2}, \ldots, t_{n} \rrbracket_{\mathrm{gr}} .
$$


Let $T$ be a torus determined by a lattice $N$, and let $\chi_{1}, \ldots, \chi_{n}$ be a basis for the dual lattice $M$. It is shown in [Krishna 2012] that the equivariant cobordism ring of a point with trivial $T$-action is isomorphic to

$$
\Omega_{T}^{*}(\operatorname{Spec} k) \cong \mathbb{L} \llbracket t_{1}, \ldots, t_{n} \rrbracket_{\mathrm{gr}},
$$

with $t_{i}$ corresponding to the first Chern class transformation $\tilde{\mathrm{c}}_{1}^{T}\left(L_{\chi_{i}}\right)$ of the equivariant line bundle $L_{\chi_{i}}$. Since this ring depends on the lattice $M$ only, we will write it as $\mathbb{\llbracket} \llbracket M \rrbracket_{\mathrm{gr}}$ (see Section 7D for a presentation of $\Omega_{T}^{*}(\operatorname{Spec} k) \cong \mathbb{L} \llbracket M \rrbracket_{\mathrm{gr}}$ functorial in $M$ and independent of the choice of a basis for $M$ ).

We will need a slight generalization of the isomorphism (7-1). First recall that, given a closed subgroup $H \subset G$, there is a natural change of groups homomorphism $\tau: \Omega_{G}^{*}(X) \rightarrow \Omega_{H}^{*}(X)$ for any smooth $X$ in $G-\operatorname{Sch}_{k}$.

Lemma 7.1. Let $X$ be a smooth scheme in $G-\mathrm{Sch}_{k}$, and let $T$ act trivially on $X$. Then there is an isomorphism

$$
\Omega_{G \times T}^{*}(X) \cong \Omega_{G}^{*}(X) \hat{\otimes}_{\mathbb{L}} \mathbb{\llbracket} M \rrbracket_{\mathrm{gr}}=\Omega_{G}^{*}(X) \llbracket M \rrbracket_{\mathrm{gr}} .
$$

This isomorphism is compatible with lci pullbacks and change of groups in the following sense. Let $f: Y \rightarrow X$ be an lci morphism between smooth schemes in $G$-Sch $k$ and let $H \subset G$ be a closed subgroup. Then the following diagrams commute:
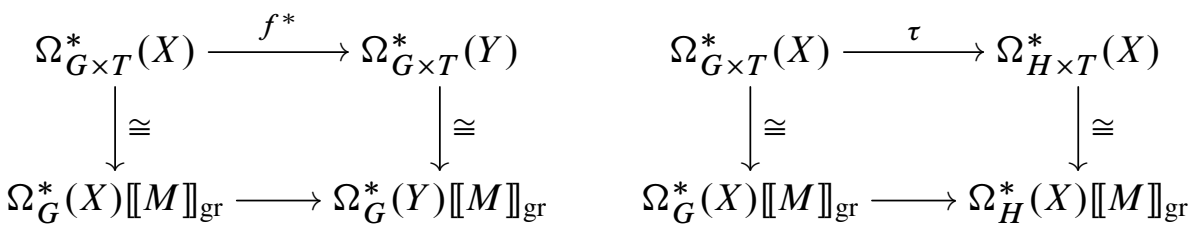

Here the lower horizontal maps are the maps induced on the tensor product by the identity on $\mathbb{\llbracket M} \rrbracket_{\mathrm{gr}}$ and the maps $f^{*}, \tau$ on the other factor. (Equivalently, the maps are $f^{*}$ and $\tau$ applied to the coefficients of power series.)

Proof. We may assume that $\operatorname{dim} T=1$. Let

$$
\Omega_{G}^{*}(X)=\lim _{j} \Omega^{*}\left(X \times{ }^{G} U_{j}\right),
$$

where $\left\{\left(V_{j}, U_{j}\right)\right\}$ is a good system of representations of $G$. Let $T$ act on $\mathbb{A}^{j}=k^{j}$ diagonally. Then we may choose $\left\{\left(\mathbb{A}^{j}, \mathbb{A}^{j} \backslash\{0\}\right)\right\}$ as a good system of representations for $T$. Now,

$$
\begin{aligned}
\Omega_{G \times T}^{*}(X) & =\varlimsup_{j} \Omega^{*}\left(X \times{ }^{G \times T}\left(U_{j} \times \mathbb{A}^{j} \backslash\{0\}\right)\right) \\
& =\varlimsup_{j} \Omega^{*}\left(\left(X \times{ }^{G} U_{j}\right) \times \mathbb{P}^{j-1}\right) \\
& =\longleftarrow_{j} \Omega^{*}\left(X \times{ }^{G} U_{j}\right) \otimes_{\mathbb{L}} \mathbb{L}[t] /\left(t^{j}\right) \\
& =\Omega_{G}^{*}(X) \hat{\otimes}_{\mathbb{L}} \mathbb{L} \llbracket t \rrbracket_{\mathrm{gr}} .
\end{aligned}
$$


In the third equality we used the projective bundle formula [Levine and Morel 2007]

$$
\Omega^{*}\left(Z \times \mathbb{P}^{n}\right) \cong \Omega^{*}(Z)[t] /\left(t^{n+1}\right),
$$

for any smooth scheme $Z$. The compatibility of each step with the change of group homomorphisms and the compatibility of the projective bundle isomorphism with lci pullbacks $W \rightarrow Z$ give the compatibility statement of the lemma.

7B. Piecewise graded power series on $\Delta$. Consider now a toric variety $X_{\Delta}$. There is a one-to-one correspondence between cones $\sigma \in \Delta$ and $T$-orbits in $X_{\Delta}$. Let $O_{\sigma}$ be the orbit corresponding to a cone $\sigma$. Then the stabilizer (of a point) of $O_{\sigma}$ is the subtorus $T_{\sigma} \subset T$ corresponding to the sublattice $N_{\sigma}=\operatorname{Span} \sigma \cap N \subset N$. The Morita isomorphism [Krishna and Uma 2013] then gives

$$
\Omega_{T}^{*}\left(O_{\sigma}\right)=\Omega_{T}^{*}\left(T \times{ }^{T_{\sigma}} \operatorname{Spec} k\right) \cong \Omega_{T_{\sigma}}^{*}(\operatorname{Spec} k) .
$$

We set $S_{\sigma}=\Omega_{T}^{*}\left(O_{\sigma}\right) \cong \Omega_{T_{\sigma}}^{*}(\operatorname{Spec} k)$. When $\tau$ is a face of $\sigma$, we have the inclusion of lattices $N_{\tau} \subset N_{\sigma}$, giving rise to the inclusion of tori $T_{\tau} \subset T_{\sigma}$; the change of groups homomorphism then defines the restriction map $S_{\sigma} \rightarrow S_{\tau}$. Let $S=\Omega_{T}^{*}(\operatorname{Spec} k)$. The rings $S_{\sigma}$ and $S_{\tau}$ are graded $S$-algebras and the restriction map $S_{\sigma} \rightarrow S_{\tau}$ is a morphism of graded $S$-algebras.

A piecewise graded power series on $\Delta$ is a collection $\left(a_{\sigma} \in S_{\sigma}\right)_{\sigma \in \Delta}$ such that $a_{\sigma}$ restricts to $a_{\tau}$ whenever $\sigma \geq \tau$. Let $\operatorname{PPS}(\Delta)$ be the graded $S$-algebra of all piecewise graded power series on $\Delta$. Similarly, let $\operatorname{PPS}(\operatorname{St} \rho)$ be the graded $S$ algebra of piecewise graded power series on St $\rho=\{\sigma \in \Delta \mid \rho \leq \sigma\}$, that is, collections $\left(a_{\sigma} \in S_{\sigma}\right)_{\sigma \in \mathrm{St} \rho}$ such that $a_{\sigma}$ restricts to $a_{\tau}$ for $\sigma \geq \tau \in \mathrm{St} \rho$.

7C. Operational equivariant cobordism of toric varieties. The inclusion map $i_{\sigma}$ : $O_{\sigma} \hookrightarrow X_{\Delta}$ is an lci morphism when $X_{\Delta}$ is smooth; hence there exists a pullback map

$$
i_{\sigma}^{*}: \Omega_{T}^{*}\left(X_{\Delta}\right) \rightarrow \Omega_{T}^{*}\left(O_{\sigma}\right)=S_{\sigma} .
$$

Theorem 7.2 [Krishna and Uma 2013]. Let $X_{\Delta}$ be a smooth quasiprojective toric variety. Then the morphism of $S$-algebras

$$
\Omega_{T}^{*}\left(X_{\Delta}\right) \stackrel{\left(i_{\sigma}^{*}\right)}{\longrightarrow} \prod_{\sigma \in \Delta} S_{\sigma}
$$

is injective and the image is equal to the $S$-algebra $\operatorname{PPS}(\Delta)$ of piecewise graded power series on $\Delta$.

In the proof of Krishna and Uma, the group $\Omega_{T}^{*}\left(X_{\Delta}\right)$ stands for the cohomological notation of $\Omega_{\operatorname{dim} X_{\Delta-*}}^{T}\left(X_{\Delta}\right)$ and the maps $i_{\sigma}^{*}$ are lci pullbacks. We claim that the same statement is true for general $X_{\Delta}$ when $\Omega_{T}^{*}\left(X_{\Delta}\right)$ stands for the operational cobordism ring and $i_{\sigma}^{*}$ is the pullback morphism in the operational theory. 
Theorem 7.3. Let $X_{\Delta}$ be a quasiprojective toric variety. Then the morphism of $S$-algebras

$$
\Omega_{T}^{*}\left(X_{\Delta}\right) \stackrel{\left(i_{\sigma}^{*}\right)}{\longrightarrow} \prod_{\sigma \in \Delta} S_{\sigma}
$$

is injective and the image is equal to the $S$-algebra $\operatorname{PPS}(\Delta)$ of piecewise graded power series on $\Delta$.

Proof. We prove the theorem by induction on $\operatorname{dim} X_{\Delta}$. In the inductive proof we will need a slightly stronger statement. Let $\widetilde{T}$ be another torus and $\widetilde{T} \rightarrow T$ a split surjective group homomorphism $\widetilde{T} \cong T \times T^{\prime}$ from some torus $T^{\prime}$. Let $\widetilde{T}$ act on $X_{\Delta}$ via the homomorphism $\tilde{T} \rightarrow T$. Replacing $T$ with $\tilde{T}$, we define as above $\widetilde{S}=\Omega_{\widetilde{T}}^{*}(\operatorname{Spec} k), \widetilde{S}_{\sigma}=\Omega_{\widetilde{T}}^{*}\left(O_{\sigma}\right)$, and the restriction maps $\widetilde{S}_{\sigma} \rightarrow \widetilde{S}_{\tau}$ for $\sigma \geq \tau$ (note that $T$ and $\widetilde{T}$ have the same orbits). We let $\widetilde{\operatorname{PPS}}(\Delta)$ denote the $\widetilde{S}$ algebra of piecewise graded power series on $\Delta$ defined using the rings $\widetilde{S}_{\sigma}$. The $\widetilde{S}$ algebra $\widetilde{\mathrm{PPS}}(\mathrm{St} \rho)$ is defined similarly.

Instead of the theorem, we prove the following stronger statement:

Claim. The morphism

$$
\Omega_{\widetilde{T}}^{*}\left(X_{\Delta}\right) \stackrel{\left(i_{\sigma}^{*}\right)}{\longrightarrow} \prod_{\sigma \in \Delta} \widetilde{S}_{\sigma}
$$

is injective and the image is equal to the graded $\widetilde{S}$-algebra $\widetilde{\operatorname{PPS}}(\Delta)$.

Let us first check the claim for smooth $X_{\Delta}$. The statement of Theorem 7.2 can be restated as saying that the following sequence is exact:

$$
0 \longrightarrow \Omega_{T}^{*}\left(X_{\Delta}\right) \stackrel{\left(i_{\sigma}^{*}\right)}{\longrightarrow} \prod_{\sigma \in \Delta} S_{\sigma} \rightrightarrows \prod_{\sigma, \tau \in \Delta} S_{\sigma \cap \tau} .
$$

Here the last two maps are constructed from restrictions $S_{\sigma} \rightarrow S_{\sigma \cap \tau}$ and $S_{\sigma} \rightarrow S_{\tau \cap \sigma}$. Let us write $\widetilde{T} \cong T \times T^{\prime}$, where $T^{\prime}$ has character lattice $M^{\prime}$. Tensoring the sequence with $\mathbb{\llbracket} \llbracket M^{\prime} \rrbracket_{\text {gr }}$, we get the exact sequence

$$
0 \rightarrow \Omega_{T}^{*}\left(X_{\Delta}\right) \llbracket M^{\prime} \rrbracket_{\mathrm{gr}} \stackrel{\left(i_{\sigma}^{*}\right)}{\longrightarrow} \prod_{\sigma \in \Delta} S_{\sigma} \llbracket M^{\prime} \rrbracket_{\mathrm{gr}} \rightrightarrows \prod_{\sigma, \tau \in \Delta} S_{\sigma \cap \tau} \llbracket M^{\prime} \rrbracket_{\mathrm{gr}} .
$$

The maps in this sequence are the old maps applied to coefficients of power series. From Lemma 7.1 we know that this sequence is isomorphic to the sequence

$$
0 \rightarrow \Omega_{\widetilde{T}}^{*}\left(X_{\Delta}\right) \stackrel{\left(i_{\sigma}^{*}\right)}{\longrightarrow} \prod_{\sigma \in \Delta} \tilde{S}_{\sigma} \rightrightarrows \prod_{\sigma, \tau \in \Delta} \tilde{S}_{\sigma \cap \tau},
$$

where the maps are again lci pullbacks and change of group homomorphisms. This proves the claim in the case of smooth $X_{\Delta}$. 
When $X_{\Delta}$ is singular, we resolve its singularities by a sequence of star subdivisions of $\Delta$ :

$$
X_{\Delta} \longleftarrow X_{\Delta^{\prime}} \longleftarrow \cdots \longleftarrow X_{\Delta^{\prime \prime}}
$$

We may assume by induction on the number of star subdivisions that the claim holds for $X_{\Delta^{\prime}}$. The morphism $f: X_{\Delta^{\prime}} \rightarrow X_{\Delta}$ is the blowup of $X_{\Delta}$ along a closed subscheme $C \subset X_{\Delta}$ with support $|C|$ equal to the orbit closure $V_{\pi}=\bar{O}_{\pi}$, where $\pi \in \Delta$ is the cone containing the subdivision ray in its relative interior. Let $\rho \in \Delta^{\prime}$ be the new ray. Then the exceptional divisor $E=f^{-1}(C)$ has support $|E|=V_{\rho}$. The morphism $f$ is a birational envelope. In order to use Theorem 6.2, we need to identify $\Omega_{\widetilde{T}}^{*}(C), \Omega_{\widetilde{T}}^{*}(E)$ and the pullback map between them.

Lemma 7.4. For any $0 \neq \pi \in \Delta$, the map

$$
\Omega_{\widetilde{T}}^{*}\left(V_{\pi}\right) \stackrel{\left(i_{\sigma}^{*}\right)}{\longrightarrow} \prod_{\sigma \in \operatorname{St}(\pi)} \widetilde{S}_{\sigma}
$$

is injective and the image is equal to the graded $\widetilde{S}$-algebra $\widetilde{\operatorname{PPS}}(\mathrm{St} \pi)$.

Proof. The orbit closure $V_{\pi}$ is again a toric variety corresponding to the fan $\Delta_{\pi}$ that is the image of St $\pi$ in $N /(\operatorname{Span} \pi \cap N)$. There is a split surjection from the torus $T$ (and hence also from $\widetilde{T}$ ) to the big torus in $V_{\pi}$. The result now follows by induction on the dimension of the toric variety.

We can also apply the previous lemma to $\rho \in \Delta^{\prime}$, to get that $\Omega_{\widetilde{T}}^{*}\left(V_{\rho}\right)$ is isomorphic to $\widetilde{\operatorname{PPS}}(\mathrm{St} \rho)$. Moreover, since by assumption we know the claim for $X_{\Delta^{\prime}}$, the pullback map $\Omega_{\widetilde{T}}^{*}\left(X_{\Delta^{\prime}}\right) \rightarrow \Omega_{\widetilde{T}}^{*}\left(V_{\rho}\right)$ is the restriction of piecewise power series $\widetilde{\operatorname{PPS}}\left(\Delta^{\prime}\right) \rightarrow \widetilde{\operatorname{PPS}}($ St $\rho)$.

Next we describe the pullback morphism $\Omega_{\widetilde{T}}^{*}\left(V_{\pi}\right) \rightarrow \Omega_{\widetilde{T}}^{*}\left(V_{\rho}\right)$. Note that every cone $\sigma \in$ St $\rho$ lies in some cone of $\tau \in \mathrm{St} \pi$, and hence we have the restriction map $\widetilde{S}_{\tau} \rightarrow \widetilde{S}_{\sigma}$. These maps combine to give a well-defined pullback map of piecewise graded power series $\widetilde{\operatorname{PPS}}(\mathrm{St} \pi) \rightarrow \widetilde{\operatorname{PPS}}($ St $\rho)$.

Lemma 7.5. The pullback morphism

$$
\Omega_{\widetilde{T}}^{*}\left(V_{\pi}\right) \cong \widetilde{\operatorname{PPS}}(\operatorname{St} \pi) \rightarrow \Omega_{\widetilde{T}}^{*}\left(V_{\rho}\right) \cong \widetilde{\operatorname{PPS}}(\operatorname{St} \rho)
$$

is the pullback of piecewise graded power series.

Proof. Define a map $\phi: \Delta^{\prime} \rightarrow \Delta$ so that $\phi(\sigma)$ is the smallest cone in $\Delta$ containing $\sigma$. Then the map $f: X_{\Delta^{\prime}} \rightarrow X_{\Delta}$ takes $O_{\sigma}$ onto $O_{\phi(\sigma)}$. The pullback morphism

$$
\Omega_{\widetilde{T}}^{*}\left(O_{\phi(\sigma)}\right)=\widetilde{S}_{\phi(\sigma)} \rightarrow \Omega_{\widetilde{T}}^{*}\left(O_{\sigma}\right)=\tilde{S}_{\sigma}
$$

is the restriction of power series. 
Consider the commutative diagram

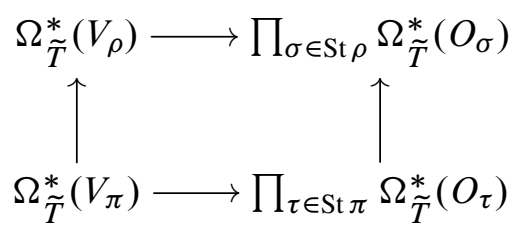

where all maps are pullback morphisms. The right vertical map sends $\left(a_{\tau}\right)$ to $\left(b_{\sigma}\right)$ such that $b_{\sigma}$ is the restriction of $a_{\phi(\sigma)}$; this map restricts to the pullback of piecewise power series $\widetilde{\operatorname{PPS}}(\mathrm{St} \pi) \rightarrow \widetilde{\operatorname{PPS}}(\mathrm{St} \rho)$, which proves the lemma.

To finish the proof of the claim, we apply Theorem 6.2 with $S_{1}=C^{\text {red }}=V_{\pi}$ and $E_{1}=E^{\text {red }}=V_{\rho}$. Since the pullback map $\Omega_{\widetilde{T}}^{*}\left(V_{\pi}\right) \rightarrow \Omega_{\widetilde{T}}^{*}\left(V_{\rho}\right)$ is injective, Theorem 6.2 implies that we have a Cartesian diagram, with all maps pullbacks:

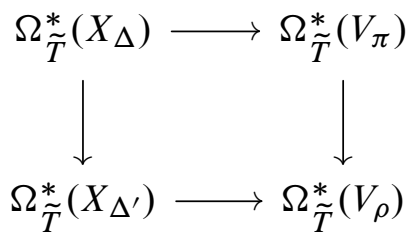

The following diagram of piecewise power series and pullback maps is clearly Cartesian:

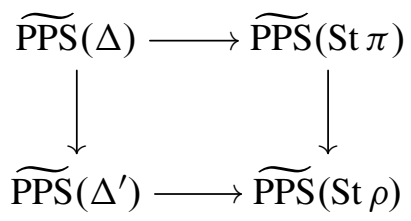

The first diagram maps to the second one by the pullback maps $i_{\sigma}^{*}$. This implies that $\Omega_{\widetilde{T}}^{*}\left(X_{\Delta}\right) \cong \widetilde{\operatorname{PPS}}(\Delta)$.

7D. Piecewise graded exponential power series. We give in this subsection a canonical presentation of the ring

$$
\Omega_{T}^{*}(\operatorname{Spec} k) \cong \mathbb{\llbracket} M \rrbracket_{\mathrm{gr}}
$$

that is functorial in $M$ and independent of the choice of a basis for $M$. This leads to the description of $\Omega_{T}^{*}\left(X_{\Delta}\right)$ as the algebra of piecewise graded exponential power series, similar to the case of equivariant $K$-theory [Anderson and Payne 2015]. An even more general construction of the formal group ring $R \llbracket M \rrbracket_{F}$ was given in [Calmès et al. 2013] for an arbitrary ring $R$ with a formal group law $F$. 
Let $M$ be the character lattice of a torus $T$, and define

$$
\mathbb{\llbracket} \llbracket M \rrbracket_{\mathrm{gr}}=\bigoplus_{d} \prod_{k} \mathbb{1}_{d-k} \otimes \operatorname{Sym}^{k} M .
$$

This ring is (noncanonically) isomorphic to the graded power series $\operatorname{ring}$ in $\operatorname{rank}(M)$ variables.

For any formal group law $F$ on a ring $R$ there exists a unique power series

$$
e_{F}(x)=x+b_{2} x^{2}+b_{3} x^{3}+\cdots \in R \llbracket x \rrbracket_{\mathrm{gr}} \otimes \mathbb{Q},
$$

called the exponential series, such that

$$
F\left(e_{F}(u), e_{F}(v)\right)=e_{F}(u+v) .
$$

The series $e_{F}(x)$ is homogeneous of degree 1. (See [Levine and Morel 2007, Lemma 4.1.29] for the construction of the inverse power series $l_{F}(x)$.) For the additive group law $F(u, v)=u+v$, we have $e_{F}(x)=x$. For the multiplicative group law $F(u, v)=u+v+b u v$,

$$
e_{F}(x)=x+b \frac{x^{2}}{2 !}+b^{2} \frac{x^{3}}{3 !}+\cdots
$$

We consider the exponential power series $e(x)$ for the formal group law $F_{\mathbb{L}}$ on $\mathbb{L}$. The map

$$
e: M \rightarrow \mathbb{\llbracket} \llbracket M \rrbracket_{\mathrm{gr}} \otimes \mathbb{Q}
$$

that sends $u$ to $e(u)$ satisfies the equality $F_{\mathbb{L}}(e(u), e(v))=e(u+v)$. We get a canonical isomorphism

$$
\mathbb{L} \llbracket M \rrbracket_{\mathrm{gr}} \otimes \mathbb{Q} \cong \Omega_{T}(\operatorname{Spec} k) \otimes \mathbb{Q},
$$

identifying $e(u)$ with the first Chern class transformation $\tilde{\mathrm{c}}_{1}^{T}\left(L_{u}\right)$. The integral cobordism ring $\Omega_{T}$ (Spec $k$ ) is then canonically isomorphic to the subring of $\mathbb{L} \llbracket M \rrbracket_{\text {gr }} \otimes \mathbb{Q}$ consisting of graded power series in $e(u)$ for $u \in M$ and coefficients in $\mathbb{L}$. (Here we need the fact that the additive group $\mathbb{L}$ is a free abelian group and hence embeds in $\mathbb{L} \otimes \mathbb{Q}$.)

The construction of the ring of graded exponential power series is functorial in $M$. Indeed, a homomorphism of lattices $M \rightarrow M^{\prime}$ gives rise to the ring homomorphism $\mathbb{L} \llbracket M \rrbracket_{\mathrm{gr}} \rightarrow \mathbb{L} \llbracket M^{\prime} \rrbracket_{\mathrm{gr}}$, such that the diagram

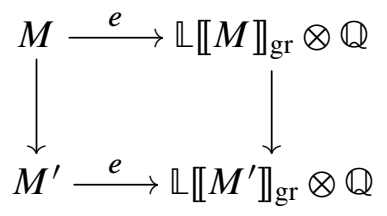


is commutative. It follows that graded exponential power series are mapped to graded exponential power series. This corresponds to the pullback map

$$
\Omega_{T}(\operatorname{Spec} k) \rightarrow \Omega_{T^{\prime}}(\operatorname{Spec} k) .
$$

Theorem 7.3 now states that the equivariant operational cobordism ring of $X_{\Delta}$ is canonically isomorphic to the ring of piecewise graded exponential power series on $\Delta$.

Acknowledgments. The authors would like to thank Dave Anderson, William Fulton and Sam Payne for insightful conversations. Sam Payne has been very helpful in explaining to us why the quasiprojectivity requirement can be relaxed in defining $T$-equivariant theories. We also thank the anonymous reviewer for the very useful feedback and for explaining how to make the $T$-equivariant cobordism ring of a point canonically isomorphic to a ring of graded power series (see Section 7D). This work started by trying to understand the operational equivariant $K$-theory of toric varieties constructed by Dave Anderson and Sam Payne.

\section{References}

[Anderson and Payne 2015] D. Anderson and S. Payne, "Operational K-theory", Doc. Math. 20 (2015), 357-399.

[Borel 1991] A. Borel, Linear algebraic groups, 2nd ed., Graduate Texts in Mathematics 126, Springer, New York, 1991. MR 92d:20001 Zbl 0726.20030

[Brion 1997] M. Brion, "Equivariant Chow groups for torus actions", Transform. Groups 2:3 (1997), 225-267. MR 99c:14005 Zbl 0916.14003

[Brion and Vergne 1997] M. Brion and M. Vergne, "An equivariant Riemann-Roch theorem for complete, simplicial toric varieties”, J. Reine Angew. Math. 482 (1997), 67-92. MR 98a:14067 Zbl 0842.73005

[Calmès et al. 2013] B. Calmès, V. Petrov, and K. Zainoulline, "Invariants, torsion indices and oriented cohomology of complete flags", Ann. Sci. Éc. Norm. Supér. (4) 46:3 (2013), 405-448. MR 3099981 Zbl 06216390

[Deshpande 2009] D. Deshpande, “Algebraic cobordism of classifying spaces”, preprint, 2009. arXiv 0907.4437

[Edidin and Graham 1998] D. Edidin and W. Graham, "Equivariant intersection theory", Invent. Math. 131:3 (1998), 595-634. MR 99j:14003a Zbl 0940.14003

[Fulton 1993] W. Fulton, Introduction to toric varieties, Annals of Mathematics Studies 131, Princeton University Press, 1993. MR 94g:14028 Zbl 0813.14039

[Fulton 1998] W. Fulton, Intersection theory, 2nd ed., Ergebnisse der Mathematik und ihrer Grenzgebiete (3) 2, Springer, Berlin, 1998. MR 99d:14003 Zbl 0885.14002

[Fulton and MacPherson 1981] W. Fulton and R. MacPherson, Categorical framework for the study of singular spaces, Mem. Amer. Math. Soc. 31, Amer. Math. Soc., Providence, RI, 1981. MR 83a:55015 Zbl 0467.55005

[Gillet 1984] H. Gillet, "Homological descent for the $K$-theory of coherent sheaves", pp. 80-103 in Algebraic K-theory, number theory, geometry and analysis (Bielefeld, 1982), edited by A. Bak, Lecture Notes in Math. 1046, Springer, Berlin, 1984. MR 86a:14016 Zbl 0862.14006 
[González and Karu 2015] J. L. González and K. Karu, "Descent for algebraic cobordism", J. Algebraic Geom. 24 (2015), 787-804.

[Heller and Malagón-López 2013] J. Heller and J. Malagón-López, "Equivariant algebraic cobordism”, J. Reine Angew. Math. 684 (2013), 87-112. MR 3181557 Zbl 06237587

[Kimura 1992] S.-i. Kimura, "Fractional intersection and bivariant theory", Comm. Algebra 20:1 (1992), 285-302. MR 93d:14010 Zbl 0774.14004

[Krishna 2012] A. Krishna, "Equivariant cobordism of schemes", Doc. Math. 17 (2012), 95-134. MR 2889745 Zbl 1246.14016

[Krishna and Uma 2013] A. Krishna and V. Uma, "The algebraic cobordism ring of toric varieties", Int. Math. Res. Not. 2013:23 (2013), 5426-5464. MR 3142260

[Levine and Morel 2007] M. Levine and F. Morel, Algebraic cobordism, Springer, Berlin, 2007. MR 2008a:14029 Zbl 1188.14015

[Levine and Pandharipande 2009] M. Levine and R. Pandharipande, "Algebraic cobordism revisited", Invent. Math. 176:1 (2009), 63-130. MR 2010h:14033 Zbl 1210.14025

[Mumford et al. 1994] D. Mumford, J. Fogarty, and F. Kirwan, Geometric invariant theory, 3rd ed., Ergebnisse der Mathematik und ihrer Grenzgebiete (2) 34, Springer, Berlin, 1994. MR 95m:14012 Zbl 0797.14004

[Payne 2006] S. Payne, "Equivariant Chow cohomology of toric varieties", Math. Res. Lett. 13:1 (2006), 29-41. MR 2007f:14052 Zbl 1094.14036

[Totaro 1999] B. Totaro, "The Chow ring of a classifying space", pp. 249-281 in Algebraic K-theory (Seattle, WA, 1997), edited by W. Raskind and C. Weibel, Proc. Sympos. Pure Math. 67, Amer. Math. Soc., Providence, RI, 1999. MR 2001f:14011 Zbl 0967.14005

[Vezzosi and Vistoli 2003] G. Vezzosi and A. Vistoli, "Higher algebraic $K$-theory for actions of diagonalizable groups", Invent. Math. 153:1 (2003), 1-44. MR 2006c:19003 Zbl 1032.19001

[Yokura 2009] S. Yokura, "Oriented bivariant theories, I", Internat. J. Math. 20:10 (2009), 1305-1334. MR 2011d:55010 Zbl 1195.55006

Communicated by Mikhail Kapranov

Received 2013-01-28 Revised 2015-04-21 Accepted 2015-05-20

jose.gonzalez@yale.edu Department of Mathematics, Yale University, 10 Hillhouse Avenue, New Haven, CT 06511, United States

karu@math.ubc.ca

Department of Mathematics, University of British Columbia, 1984 Mathematics Road, Vancouver, BC V6T 1Z2, Canada 


\section{Algebra \& Number Theory}

msp.org/ant

\section{EDITORS}

MANAGING EDITOR

Bjorn Poonen

Massachusetts Institute of Technology

Cambridge, USA

\author{
EDITORIAL BOARD CHAIR \\ David Eisenbud \\ University of California \\ Berkeley, USA
}

BOARD OF EDITORS

Georgia Benkart

Dave Benson

Richard E. Borcherds

John H. Coates

J-L. Colliot-Thélène

Brian D. Conrad

Hélène Esnault

Hubert Flenner

Sergey Fomin

Edward Frenkel

Andrew Granville

Joseph Gubeladze

Roger Heath-Brown

Craig Huneke

Kiran S. Kedlaya

János Kollár

Yuri Manin

Philippe Michel
University of Wisconsin, Madison, USA

University of Aberdeen, Scotland

University of California, Berkeley, USA

University of Cambridge, UK

CNRS, Université Paris-Sud, France

Stanford University, USA

Freie Universität Berlin, Germany

Ruhr-Universität, Germany

University of Michigan, USA

University of California, Berkeley, USA

Université de Montréal, Canada

San Francisco State University, USA

Oxford University, UK

University of Virginia, USA

Univ. of California, San Diego, USA

Princeton University, USA

Northwestern University, USA

École Polytechnique Fédérale de Lausanne
Susan Montgomery

Shigefumi Mori

Raman Parimala

Jonathan Pila

Anand Pillay

Victor Reiner

Peter Sarnak

Joseph H. Silverman

Michael Singer

Vasudevan Srinivas

J. Toby Stafford

Ravi Vakil

Michel van den Bergh

Marie-France Vignéras

Kei-Ichi Watanabe

Efim Zelmanov

Shou-Wu Zhang
University of Southern California, USA

RIMS, Kyoto University, Japan

Emory University, USA

University of Oxford, UK

University of Notre Dame, USA

University of Minnesota, USA

Princeton University, USA

Brown University, USA

North Carolina State University, USA

Tata Inst. of Fund. Research, India

University of Michigan, USA

Stanford University, USA

Hasselt University, Belgium

Université Paris VII, France

Nihon University, Japan

University of California, San Diego, USA

Princeton University, USA

\section{PRODUCTION}

production@msp.org

Silvio Levy, Scientific Editor

See inside back cover or msp.org/ant for submission instructions.

The subscription price for 2015 is US $\$ 255 /$ year for the electronic version, and $\$ 440 /$ year ( $+\$ 55$, if shipping outside the US) for print and electronic. Subscriptions, requests for back issues and changes of subscribers address should be sent to MSP.

Algebra \& Number Theory (ISSN 1944-7833 electronic, 1937-0652 printed) at Mathematical Sciences Publishers, 798 Evans Hall \#3840, c/o University of California, Berkeley, CA 94720-3840 is published continuously online. Periodical rate postage paid at Berkeley, CA 94704, and additional mailing offices.

ANT peer review and production are managed by EditFLOW ${ }^{\circledR}$ from MSP.

\section{PUBLISHED BY}

- mathematical sciences publishers

nonprofit scientific publishing

http://msp.org/

(C) 2015 Mathematical Sciences Publishers 


\section{Algebra \& Number Theory}

Volume $9 \quad$ No. $6 \quad 2015$

Bivariant algebraic cobordism

José LUIS GONZÁLEZ and KALLE KARU

Schubert decompositions for quiver Grassmannians of tree modules OLIVER LORSCHEID

Noncommutative geometry and Painlevé equations

ANDREI OKOUNKOV and ERIC RAINS

Electrical networks and Lie theory

ThOMas Lam and Pavlo PylyavskyY

The Kac-Wakimoto character formula for the general linear Lie superalgebra Michael Chmutov, Crystal Hoyt and ShifRa Reif

Effective Matsusaka's theorem for surfaces in characteristic $p$

Gabriele Di Cerbo and ANDREA FANELli

Adams operations and Galois structure

GEORGIOS PAPPAS 